\title{
Lyapunov functions to Caputo reaction-diffusion fractional neural networks with time-varying delays
}

\author{
R. P. Agarwala,b,*, S. Hristovac ${ }^{\mathrm{c}}$, D. O’Regan ${ }^{\mathrm{d}}$ \\ a Department of Mathematics, Texas A\&M University-Kingsville, Kingsville, TX 78363, USA. \\ ${ }^{b}$ Distinguished University Professor of Mathematics, Florida Institute of Technology, Melbourne, FL 32901, USA. \\ ${ }^{c}$ University of Plovdiv Paisii Hilendarski, Plovdiv, Bulgaria. \\ ${ }^{d}$ School of Mathematics, Statistics and Applied Mathematics, National University of Ireland, Galway, Ireland.
}

\begin{abstract}
A reaction diffusion equation with a Caputo fractional derivative in time and with time-varying delays is considered. Stability properties of the solutions are studied via the direct Lyapunov method and arbitrary Lyapunov functions (usually quadratic Lyapunov functions are used). In this paper we give a brief overview of the most popular fractional order derivatives of Lyapunov functions among Caputo fractional delay differential equations. These derivatives are applied to various types of reaction-diffusion fractional neural network with variable coefficients and time-varying delays. We show the quadratic Lyapunov functions and their Caputo fractional derivatives are not applicable in some cases when one studies stability properties. Some sufficient conditions for stability are obtained and we illustrate our theory on a particular nonlinear Caputo reaction-diffusion fractional neural network with time dependent delays.
\end{abstract}

Keywords: Reaction-diffusion fractional neural networks, delays, Caputo derivatives, Lyapunov functions, stability, fractional derivative of Lyapunov functions.

2010 MSC: 35R11, 34K37, 34K20.

(C)2018 All rights reserved.

\section{Introduction}

Neural networks have a wide range of applications in pattern recognition, associative memory, combinatorial optimization, etc.. Delays are incorporated into the model equations of the networks because of the finite speeds of the switching and the transmission of signals in a network and this leads to time delays in a working network. It was observed both experimentally and numerically in [18] that time delay could induce instability, causing sustained oscillations which may be harmful to a system. Additionally, when one considers memory and hereditary properties of various materials and processes ([9]) it is natural to consider fractional derivatives in the model. Neural networks in biology, coupled lasers,

\footnotetext{
*Corresponding author

Email address: Ravi.Agarwal@tamuk.edu (R. P. Agarwal)

doi: $10.22436 /$ jmcs.018.03.08
}

Received: 2017-11-05 Revised: 2018-03-12 Accepted: 2018-04-20 
wireless communication, and power-grid networks in physics and engineering $([19,27,29])$ are modeled by fractional order differential equations.

In controlling nonlinear systems, the Lyapunov second method provides a way to analyze the stability of the system without explicitly solving the differential equations. Stability results concerning integerorder neural networks can be found in $[11,15,31]$ and recently Lyapunov stability theory for fractional order systems was discussed (see [1, 3-5, 13, 14]). Fractional order Lyapunov stability theory was studied for various types of fractional neural networks using quadratic Lyapunov functions (see, for example, [33]) and stability analysis of fractional-order delay neural networks can be found in for example, $[6-8,30,32]$ and the references therein. Space-time fractional reaction-diffusion equations with Riemann-Liouville fractional Derivative is studied in [23].

In this paper we consider Caputo fractional derivatives with order $q \in(0,1)$ defined by (see, for example, [20])

$$
{ }_{\mathfrak{t}_{0}}^{\mathrm{C}} D_{\mathrm{t}}^{\mathrm{q}} \mathrm{m}(\mathrm{t})=\frac{1}{\Gamma(1-\mathrm{q})} \int_{\mathrm{t}_{0}}^{\mathrm{t}}(\mathrm{t}-\mathrm{s})^{-\mathrm{q}} \mathrm{m}^{\prime}(\mathrm{s}) \mathrm{ds}, \quad \mathrm{t} \in\left(\mathrm{t}_{0}, \mathrm{t}_{0}+\mathrm{T}\right),
$$

where $\Gamma$ (.) denotes the Gamma function, $\mathrm{t}_{0} \geqslant 0, \Delta \subset \mathbb{R}^{n}, 0 \in \Delta, 0<\mathrm{T} \leqslant \infty$ and the Riemann-Liouville fractional derivative of order $\mathrm{q} \in(0,1)$ is given by (see, for example, [20])

$$
{ }_{t_{0}}^{R L} D_{t}^{q} m(t)=\frac{1}{\Gamma(1-q)} \frac{d}{d t} \int_{t_{0}}^{t}(t-s)^{-q} m(s) d s, t \geqslant t_{0}
$$

Similar to (1.1) for any continuously differentiable function $u(t, x), t \in\left[t_{0}, t_{0}+T\right), x \in \Delta$, the time Caputo fractional derivatives with order $q \in(0,1)$ are defined by

$$
{ }_{t_{0}}^{C} D_{t}^{q} u(t, x)=\frac{1}{\Gamma(1-q)} \int_{t_{0}}^{t}(t-s)^{-q} \frac{\partial u(s, x)}{\partial s} d s, \quad t \in\left(t_{0}, t_{0}+T\right), x \in \Delta .
$$

Remark 1.1. Note in some papers the time Caputo fractional derivatives with order $q \in(0,1)$ is denoted by $\frac{\partial^{q} u(t, x)}{\partial t^{q}}$.

Lemma 1.2 ([10]). Let $\mathrm{P} \in \mathbb{R}^{\mathrm{n} \times \mathrm{n}}$ be constant, symmetric, and positive definite matrix and $\mathrm{m}(\mathrm{t}): \mathbb{R}_{+} \rightarrow \mathbb{R}^{\mathrm{n}}$ be a function with the Caputo fractional derivative existing. Then $\frac{1}{2}{ }_{0}^{C} D_{t}^{q}\left(m^{\top}(t) P m(t)\right) \leqslant m^{\top}(t) P{ }_{0}^{C} D_{t}^{q} m(t), t \geqslant 0$.

In this paper we present various definitions of fractional order derivatives of Lyapunov functions among Caputo fractional differential equations with variable delays, and we compare their application on several examples and demonstrate their advantages and disadvantages (Section 2). Then fractional order Lyapunov stability theory is proposed to Caputo reaction-diffusion fractional neural networks with time-varying delays. Some stability sufficient criteria using the appropriate fractional derivative of Lyapunov functions are provided and illustrated with examples on several types of Caputo reaction-diffusion fractional neural network with time-varying delays (Section 3).

\section{Fractional derivatives of Lyapunov functions}

2.1. Brief overview of the derivative of Lyapunov functions among the delay Caputo fractional differential equations

We first consider the derivative of Lyapunov functions among the nonlinear Caputo fractional delay differential equation (FrDDE)

$$
{ }_{t_{0}}^{C} D_{t}^{q} x(t)=f\left(t, x_{t}\right) \text { for } t \in\left[t_{0}, t_{0}+T\right), \quad x\left(t_{0}+\Theta\right)=\psi(\Theta), \Theta \in[-r, 0],
$$


where $x \in \mathbb{R}^{n}, f \in C\left[\left[t_{0}, t_{0}+T\right) \times \mathbb{R}^{n}, \mathbb{R}^{n}\right], t_{0} \in \mathbb{R}_{+}$is the initial time, $\psi \in C\left([-r, 0], \mathbb{R}^{n}\right)$ is the initial function, $T \leqslant \infty$, and for any $t \in\left[t_{0}, t_{0}+T\right)$ the notation $x_{t}(\Theta)=x(t+\Theta), \Theta \in[-r, 0]$ is used.

Let $x(t), t \in\left[t_{0}-r, t_{0}+T\right)$, be a solution of the IVP for the FrDDE (2.1) and let $V(t, x)$ be a Lyapunov function, i.e., $V(t, x):\left[t_{0}-r, t_{0}+T\right) \times \Delta \rightarrow \mathbb{R}_{+}$is continuous and locally Lipschitzian with respect to its second argument, where $\Delta \subset \mathbb{R}^{n}, 0 \in \Delta$.

In the literature there are three types of derivatives of Lyapunov functions among solutions of fractional differential equations used to study stability properties:

First type: Let $x(t)$ be a solution of IVP for FrDDE (2.1). Then the Caputo fractional derivative of the Lyapunov function $V(t, x(t))$ is defined by

$$
{ }_{\mathfrak{t}_{0}}^{\mathrm{C}} D_{\mathrm{t}}^{\mathrm{q}} \mathrm{V}(\mathrm{t}, x(\mathrm{t}))=\frac{1}{\Gamma(1-\mathrm{q})} \int_{\mathrm{t}_{0}}^{\mathrm{t}}(\mathrm{t}-\mathrm{s})^{-\mathrm{q}} \frac{\mathrm{d}}{\mathrm{ds}}(\mathrm{V}(\mathrm{s}, x(s))) \mathrm{ds}, \quad \mathrm{t} \in\left(\mathrm{t}_{0}, \mathrm{t}_{0}+\mathrm{T}\right) .
$$

This type of derivative is applicable for continuously differentiable Lyapunov functions. It is used mainly for quadratic Lyapunov functions to study several stability properties of fractional differential equations (see, for example, [14]).

Second type: This type of derivative of $\mathrm{V}(\mathrm{t}, \mathrm{x})$ among FrDDE (2.1) was introduced in [22, Definition 4.4] and later given in [26, Definition 1.12]:

$$
D_{(2.1)}^{+} V(t, \phi(0))=\limsup _{h \rightarrow 0} \frac{1}{h^{q}}\left[V(t, \phi(0))-V\left(t-h, \phi(0)-h^{q} f\left(t, \phi_{0}\right)\right)\right], \quad t \in\left(t_{0}, t_{0}+T\right),
$$

where $\phi \in C\left([-r, 0], \mathbb{R}^{n}\right)$ and the notation $\phi_{0}(\Theta)=\phi(0+\Theta), \Theta \in[-r, 0]$ is used.

Note the operator defined by (2.3) has no memory (memory is typical for fractional derivatives).

Remark 2.1. Let $x(t)$ be a solution of $\operatorname{FrDDE}(2.1)$ and for a fixed $t \geqslant t_{0}: \phi(s)=x(t+s), s \in[-r, 0]$. Then in general $D_{(2.1)}^{+} V(t, x(t)) \neq{ }_{t_{0}}^{C} D_{t}^{q} V(t, x(t))$ where $x(t)$ is a solution of (2.1).

Now, let us recall the remark in [28] concerning (2.3) where $V\left(t-h, \phi(0)-h^{q} f\left(t, \phi_{0}\right)\right)$ is defined by

$$
V\left(t-h, \phi(0)-h^{q} f\left(t, \phi_{0}\right)\right)=\sum_{r=1}^{\left[\frac{t-t_{0}}{h}\right]}(-1)^{r+1}{ }_{q} C_{r} V\left(t-r h, \phi(0)-h^{q} f\left(t, \phi_{0}\right)\right),
$$

where ${ }_{q} C_{r}=\frac{q(q-1) \cdots(q-r+1)}{r !}, r$ is a natural number and $[m], m>0$ is the integer part of the number $m$.

Following this notation the fractional derivative of the Lyapunov function is defined by

$$
\mathcal{D}_{(2.1)}^{+} \mathrm{V}\left(\mathrm{t}, \phi(0) ; \mathrm{t}_{0}\right)=\underset{\mathrm{h} \rightarrow 0}{\limsup } \frac{1}{\mathrm{~h}^{\mathrm{q}}}\left[\mathrm{V}(\mathrm{t}, \phi(0))-\sum_{\mathrm{r}=1}^{\left[\frac{\mathrm{t}-\mathrm{t}_{0}}{\mathrm{~h}}\right]}(-1)^{\mathrm{r}+1}{ }_{\mathrm{q}} \mathrm{C}_{\mathrm{r}} \mathrm{V}\left(\mathrm{t}-\mathrm{rh}, \phi(0)-\mathrm{h}^{\mathrm{q}} \mathrm{f}\left(\mathrm{t}, \phi_{0}\right)\right)\right]
$$

The derivative (2.4) has memory and it depends on the initial time $t_{0}$. We will call the derivative (2.4) the Dini fractional derivative of the Lyapunov function. The Dini fractional derivative is applicable for continuous Lyapunov functions.

Remark 2.2. In the general case $\mathcal{D}_{(2.1)}^{+} \mathrm{V}\left(\mathrm{t}, \phi(0) ; \mathrm{t}_{0}\right) \neq \mathrm{D}_{(2.1)}^{+} \mathrm{V}(\mathrm{t}, \phi(0))$.

Third type: For any $\phi \in C\left([-r, 0], \mathbb{R}^{n}\right)$ the derivative of the Lyapunov function $V(t, x)$ among IVP for FrDDE (2.1) with initial point $t_{0}$ and initial function $\psi \in C\left([-r, 0], \mathbb{R}^{n}\right)$ is defined by:

$$
\begin{aligned}
\stackrel{c}{(2.1)} & D_{+}^{q} V\left(t, \phi(0) ; t_{0}, \psi\right) \\
= & \limsup _{\substack{h \rightarrow 0^{+} \\
h^{q}}}\left\{V(t, \phi(0))-V\left(t_{0}, \psi(0)\right)\right. \\
& \left.-\sum_{r=1}^{\left[\frac{t-t_{0}}{h}\right]}(-1)^{r+1}{ }_{q} C_{r}\left(V\left(t-r h, \phi(0)-h^{q} f\left(t, \phi_{0}\right)\right)-V\left(t_{0}, \psi(0)\right)\right)\right\} \text { for } t \in\left(t_{0}, t_{0}+T\right),
\end{aligned}
$$


or its equivalent to

$$
\begin{aligned}
\underset{(2.1)}{c} D_{+}^{q} V\left(t, \phi(0) ; t_{0}, \psi\right)= & \limsup _{h \rightarrow 0^{+}} \frac{1}{h^{q}}\left\{V(t, \phi(0))+\sum_{r=1}^{\left[\frac{t-t_{0}}{h}\right]}(-1)^{r}{ }_{q} C_{r} V\left(t-r h, \phi(0)-h^{q} f\left(t, \phi_{0}\right)\right)\right\} \\
& -\frac{V\left(t_{0}, \psi(0)\right)}{\left(t-t_{0}\right)^{q} \Gamma(1-q)} \text { for } t \in\left(t_{0}, t_{0}+T\right) .
\end{aligned}
$$

The derivative (2.6) depends significantly on both the fractional order $q$ and the initial data $\left(t_{0}, \psi\right)$ of IVP for FrDDE (2.1) and this type of derivative is close to the idea of the Caputo fractional derivative of a function.

We call the derivative given by (2.5) or its equivalent (2.6) the Caputo fractional Dini derivative. This type of derivative is applicable for continuous Lyapunov functions (see, for example, $[1,3,5]$ ).

Remark 2.3. The equality ${ }_{(2.1)}^{c} D_{+}^{q} V\left(t, \phi(0) ; t_{0}, \psi\right)=\mathcal{D}_{(2.1)}^{+} V\left(t, \phi(0) ; t_{0}\right)-\frac{V\left(t_{0}, \psi(0)\right)}{\left(t-t_{0}\right)^{q} \Gamma(1-q)}$ holds for any $t \in$ $\left(t_{0}, t_{0}+T\right), \phi \in C\left([-r, 0], \mathbb{R}^{n}\right)$ and for any initial data $\left(t_{0}, \psi\right) \in \mathbb{R}_{+} \times C\left([-r, 0], \mathbb{R}^{n}\right):$

$$
\begin{array}{ll}
{ }_{(2.1)}^{c} D_{+}^{q} \mathrm{~V}\left(\mathrm{t}, \phi(0) ; \mathrm{t}_{0}, \psi\right)=\mathcal{D}_{(2.1)}^{+} \mathrm{V}\left(\mathrm{t}, \phi(0) ; \mathrm{t}_{0}\right), & \text { if } \mathrm{V}\left(\mathrm{t}_{0}, \psi(0)\right)=0, \\
{ }_{(2.1)}^{\mathrm{d}} \mathrm{D}_{+}^{\mathrm{q}} \mathrm{V}\left(\mathrm{t}, \phi(0) ; \mathrm{t}_{0}, \psi\right)<\mathcal{D}_{(2.1)}^{+} \mathrm{V}\left(\mathrm{t}, \phi(0) ; \mathrm{t}_{0}\right), & \text { if } \mathrm{V}\left(\mathrm{t}_{0}, \psi(0)\right)>0 .
\end{array}
$$

Remark 2.4. Note that in the case of delays in the differential equations the derivative of the Lyapunov function is considered for functions $\phi \in C\left([-r, 0], \mathbb{R}^{n}\right)$ and points $t$ such that $V(t, \phi(0))=\sup _{s \in[-r, 0]} V(t+$ $s, \phi(s))$. This condition is called the Razumikhin condition.

We will illustrate the application of the above given derivatives on a particular Lyapunov function.

Example 2.5. Let $n=1$ and the Lyapunov function $V(t, x)=g(t) x^{2}$ where $g \in C^{1}\left(\left[t_{0}, \infty\right), \mathbb{R}_{+}\right)$.

Case 1. Let $x(t)$ be a solution of the IVP for FrDDE (2.1). Then applying Eq. (2.2) the Caputo fractional derivative of the Lyapunov function is

$$
{ }_{t_{0}}^{C} D_{t}^{q} V(t, x(t))=\frac{1}{\Gamma(1-q)} \int_{t_{0}}^{t}(t-s)^{-q} \frac{d}{d s}\left(g(s) x^{2}(s)\right) d s .
$$

In the general case the above integral is difficult to solve and also obtaining upper bounds might not be possible.

In the special case $g(t) \equiv 1$, i.e., we consider the quadratic Lyapunov function, we could apply Lemma 1.2 and obtain

$$
{ }_{t_{0}}^{C} D_{t}^{q} V(t, x(t)) \leqslant 2 x(t) \underset{t_{0}}{\stackrel{C}{C}} D_{t}^{q} x(t)=2 x(t) f\left(t, x_{t}\right)
$$

Case 2. Second type of derivative.

Case 2.1: Let the function $\phi \in C\left([-r, 0], \mathbb{R}^{n}\right)$. From formula (2.3) we obtain

$$
\begin{aligned}
D_{(2.1)}^{+} V(t, \phi(0))= & \limsup _{h \rightarrow 0} \frac{1}{h^{q}}\left[g(t)(\phi(0))^{2}-g(t-h)\left(\phi(0)-h^{q} f\left(t, \phi_{0}\right)\right)^{2}\right] \\
= & \limsup _{h \rightarrow 0} \frac{1}{h^{q}}\left[(g(t)-g(t-h)) \phi^{2}(0)\right. \\
& +2 h^{q}\left(g(t-h) \phi(0) f\left(t, \phi_{0}\right)-h^{2 q}\left(g(t-h)\left(f\left(t, \phi_{0}\right)\right)^{2}\right]\right. \\
= & \phi^{2}(0) \limsup _{h \rightarrow 0} \frac{g(t)-g(t-h)}{h} h^{1-q} \\
& +2 \phi(0) f\left(t, \phi_{0}\right) \limsup _{h \rightarrow 0} g(t-h)-\left(f\left(t, \phi_{0}\right)\right)^{2} \limsup _{h \rightarrow 0} h^{q} g(t-h)=2 \phi(0) g(t) f\left(t, \phi_{0}\right) .
\end{aligned}
$$

The derivative $D_{(2.1)}^{+} V(t, \phi(0))$ does not depend on the order $q$ of the fractional derivative. 
Case 2.3: Dini fractional derivative. For any function $\phi \in C([-r, 0], \mathbb{R})$ we apply formula (2.4) and obtain

$$
\begin{aligned}
\mathcal{D}_{(2.1)}^{+} V\left(t, \phi(0) ; t_{0}\right)= & \limsup _{h \rightarrow 0} \frac{1}{h^{q}}\left[g(t) \phi^{2}(0)-\sum_{r=1}^{\left[\frac{t-t_{0}}{h}\right]}(-1)^{r+1}{ }_{q} C_{r} g(t-r h)\left(\phi(0)-h^{q} f\left(t, \phi_{0}\right)\right)^{2}\right] \\
= & \phi^{2}(0) \limsup _{h \rightarrow 0} \frac{1}{h^{q}} \sum_{r=0}^{\left[\frac{t-t_{0}}{h}\right]}(-1)^{r}{ }_{q} C_{r} g(t-r h)+2 \phi(0) g(t) f\left(t, \phi_{0}\right) \\
& -2 \phi(0) f\left(t, \phi_{0}\right) \limsup _{h \rightarrow 0} \sum_{r=0}^{\left[\frac{t-t_{0}}{h}\right]}(-1)^{r}{ }_{q} C_{r} g(t-r h) \\
& -\left(f\left(t, \phi_{0}\right)\right)^{2} \limsup _{h \rightarrow 0} h^{q} \sum_{r=1}^{\left[\frac{t-t_{0}}{h}\right]}(-1)^{r}{ }_{q} C_{r} g(t-r h) \\
= & \phi^{2}(0){ }_{t_{0}}^{R L} D_{t}^{q} g(t)+2 \phi(0) g(t) f\left(t, \phi_{0}\right) .
\end{aligned}
$$

In the case when the function $g(t) \equiv 1$, i.e., the quadratic Lyapunov function, we get

$$
\mathcal{D}_{(2.1)}^{+} \mathrm{V}\left(\mathrm{t}, \phi(0) ; \mathrm{t}_{0}\right)=2 \phi(0) \mathrm{f}\left(\mathrm{t}, \phi_{0}\right)+\frac{(\phi(0))^{2}}{\left(\mathrm{t}-\mathrm{t}_{0}\right)^{\mathrm{q}} \Gamma(1-\mathrm{q})}
$$

The Dini fractional derivative depends on both the fractional order $q$ and initial time. Similar to fractional derivatives it has a memory.

Case 2.4: Caputo fractional Dini derivative. According to Remark 2.3 and Case 2.3 we get

$$
\stackrel{\text { c }}{c} D_{1.1}^{q} D_{+}^{q} V\left(t, \phi(0) ; t_{0}, \psi\right)=\phi^{2}(0){ }_{t_{0}}^{R L} D_{t}^{q} g(t)+2 g(t) \phi(0) f\left(t, \phi_{0}\right)-\frac{g\left(t_{0}\right) \psi^{2}(0)}{\left(t-t_{0}\right)^{q} \Gamma(1-q)} .
$$

In the special case $g(t) \equiv 1$, i.e. we consider the quadratic Lyapunov function, we obtain

$$
\stackrel{c}{(2.1)} D_{+}^{q} V(t, \phi(0) ; 0, \psi)=2 \phi(0) f\left(t, \phi_{0}\right)+\frac{(\phi(0))^{2}-(\psi(0))^{2}}{\left(t-t_{0}\right)^{q} \Gamma(1-q)} .
$$

The Caputo fractional Dini derivative depends on both the fractional order $q$ and initial data which is typical for the Caputo fractional operator.

From the literature we note that one of the sufficient conditions for stability is connected with the sign of the derivative of the Lyapunov function of the equation.

Example 2.6. Consider the IVP for the scalar linear FrDDE with $q \in(0,1)$

$$
{ }_{0}^{C} D_{t}^{q} x(t)=-\left(0.5 \frac{{ }_{0}^{R L} D^{q}\left(\sin ^{2}(t)+0.1\right)}{\sin ^{2}(t)+0.1}+0.1\right) x(t)+0.1 x(t-\pi) \text { for } t>0, \quad x(s)=1 \text { for } s \in[-\pi, 0] .
$$

Denote $G(t)=-\left(0.5 \frac{{ }_{0}^{R L} D^{q}\left(\sin ^{2}(t)+0.1\right)}{\sin ^{2}(t)+0.1}+0.1\right)$.

Case 1: Consider the quadratic Lyapunov function, i.e., $V(t, x)=x^{2}$ (it is used for fractional-order neural networks with multiple time delays in [33]). Let $x(t)$ be a solution of IVP for FrDDE (2.8) and $t>0$ be such that $V(t, x(t))=x^{2}(t)=\sup _{s \in[-\pi, t]} x^{2}(s)=\sup _{s \in[-\pi, t]} V(s, x(s))$. According to Case 1 in Example 2.5 we get

$$
{ }_{0}^{C} D_{t}^{q} V(t, x(t)) \leqslant 2 x(t){ }_{0}^{C} D_{t}^{q} x(t)=2(x(t))^{2} G(t)+0.2 x(t) x(t-\pi) \leqslant 2 x^{2}(t)(0.1+G(t)) .
$$

The sign of ${ }_{0}^{C} D_{t}^{q} V(t, x(t))$ is changeable (the graph of the function $G(t)+0.1$ for various values of $q$ is given on Figure 1). 


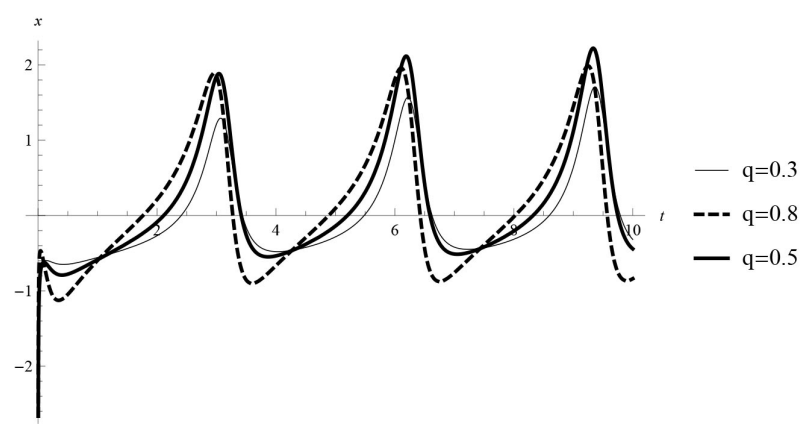

Figure 1: Example 2.6. Graph of the function $G(t)+0.1$ for various values of $\mathrm{q}$.

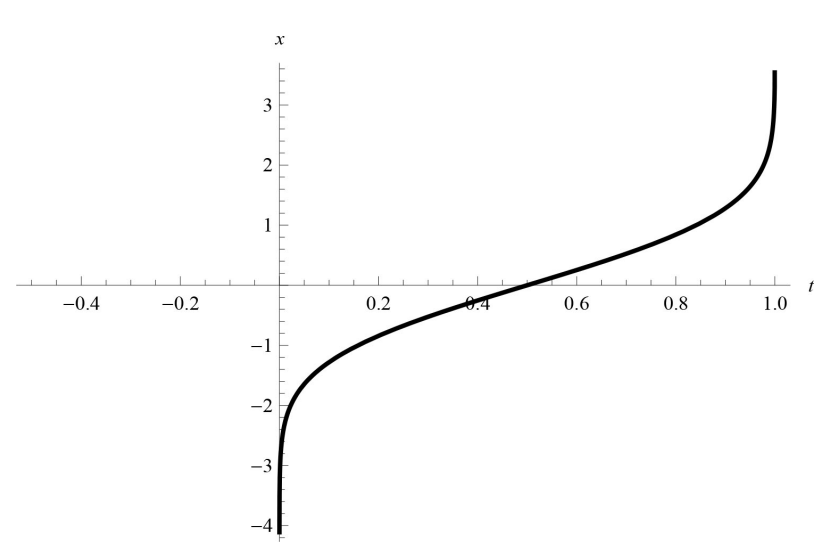

Figure 2: Example 3.5. Graph of the function $f(t)=$ $\sqrt{2} \operatorname{erf}^{-1}(2 t-1)$.

Case 2: Consider the function $V(t, x)=\left(\sin ^{2}(t)+0.1\right) x^{2}$.

Case 2.1: Caputo fractional derivative. According to Case 1 in Example 2.5 the fractional derivative of this function $\bar{V}$ is difficult to obtain so it is difficult to discuss its sign.

Case 2.2: According to Case 2.1 in Example 2.5 for any function $\phi \in \mathrm{C}([-\pi, 0], \mathbb{R})$ and any point $t>0$ such that $\mathrm{V}(\mathrm{t}, \phi(0))=\left(\sin ^{2}(\mathrm{t})+0.1\right) \phi^{2}(0)=\sup _{\mathrm{s} \in[-\pi, 0]}\left(\sin ^{2}(\mathrm{t}+\mathrm{s})+0.1\right) \phi^{2}(\mathrm{~s})=\sup _{\mathrm{s} \in[-\pi, 0]} \mathrm{V}(\mathrm{t}+\mathrm{s}, \phi(\mathrm{s}))$ we obtain

$$
\begin{aligned}
\mathrm{D}_{(2.8)}^{+} \mathrm{V}(\mathrm{t}, \phi(0)) & =2 \phi(0)\left(\sin ^{2}(\mathrm{t})+0.1\right)(\phi(0) \mathrm{G}(\mathrm{t})+0.1 \phi(-\pi)) \\
& \leqslant 2 \phi^{2}(0)\left(\sin ^{2}(\mathrm{t})+0.1\right) \mathrm{G}(\mathrm{t})+0.2 \phi(0) \phi(-\pi)\left(\sin ^{2}(\mathrm{t})+0.1\right) \\
& \leqslant 2 \phi^{2}(0)\left(\sin ^{2}(\mathrm{t})+0.1\right) \mathrm{G}(\mathrm{t})+0.1 \phi^{2}(0)\left(\sin ^{2}(\mathrm{t})+0.1\right)+0.1 \phi^{2}(-\pi)\left(\sin ^{2}(\mathrm{t}-\pi)+0.1\right) \\
& \leqslant 2 \phi^{2}(0)\left(\sin ^{2}(\mathrm{t})+0.1\right)(\mathrm{G}(\mathrm{t})+0.1),
\end{aligned}
$$

i.e., the sign of the derivative $D_{(2.8)}^{+} V(t, \phi)$ is changeable.

Case 2.3: Dini fractional derivative. According to Case 2.2 in Example 2.5 for any function $\phi \in \mathrm{C}([-\pi, 0], \mathbb{R})$ and $t>0$ such that $V(t, \phi(0))=\left(\sin ^{2}(t)+0.1\right) \phi^{2}(0)=\sup _{s \in[-\pi, 0]}\left(\sin ^{2}(t+s)+0.1\right) \phi^{2}(s)=\sup _{s \in[-\pi, 0]} V(t+$ $s, \phi(s))$ we apply formula (2.4) and obtain

$$
\mathcal{D}_{(2.8)}^{+} \mathrm{V}(\mathrm{t}, \phi(0) ; 0) \leqslant 2 \phi^{2}(0)(\mathrm{G}(\mathrm{t})+0.1)\left(\sin ^{2}(\mathrm{t})+0.1\right)+\phi^{2}(0){ }_{0}^{\mathrm{RL}} \mathrm{D}^{\mathrm{q}}\left(\sin ^{2}(\mathrm{t})+0.1\right)=0 .
$$

Case 2.4: Caputo fractional Dini derivative. According to Remark 2.3 and Case 2.3 of Example 2.5 the inequality

$$
{ }_{(2.8)}^{c} D_{+}^{q} V(t, \phi(0) ; 0, \psi)=\mathcal{D}_{(2.8)}^{+} V(t, \phi(0) ; 0)-\frac{0.1 \psi^{2}(0)}{t^{q} \Gamma(1-q)}=-\frac{0.1 \psi^{2}(0)}{t^{q} \Gamma(1-q)} \leqslant 0
$$

holds.

Therefore, for (2.8) both the Dini fractional derivative and the Caputo fractional Dini derivative seem to be more applicable than the Caputo fractional derivative of the Lyapunov function.

Remark 2.7. The above example notes that the quadratic function for studying stability properties of neural network might not be successful (especially when the right hand side depends directly on the time variable). Formula (2.3) is not appropriate for applications to fractional equations. The most general derivatives for non-homogenous fractional differential equations are Dini fractional derivatives and Caputo fractional Dini derivatives.

\subsection{Remarks on some applications of derivatives of Lyapunov functions to FrDDE.}

Some authors use the derivative defined by (2.3) to study stability properties of delay fractional differential equations $([22,24,26])$ and delayed reaction-diffusion cellular neural networks of fractional order 
([25]). The proofs are based on the following comparison result (we will give it with appropriate technical corrections).

Lemma 2.8 (Corollary 1.1 in [26]). Assume that the function $\mathrm{V} \in \mathrm{C}_{0}=\left\{\mathrm{V} \in \mathrm{C}\left(\left[\mathrm{t}_{0}, \infty\right) \times \Delta, \mathbb{R}_{+}\right), \mathrm{V}\right.$ is locally Lipschitzian in $\mathrm{x} \in \Delta\}$ and for $\mathrm{t} \geqslant \mathrm{t}_{0}$ and $\phi \in \mathrm{C}([-\mathrm{r}, 0], \Delta)$ the inequality

$$
\mathrm{D}_{(2.1)}^{+} \mathrm{V}(\mathrm{t}, \phi(0)) \leqslant \mathrm{BV}(\mathrm{t}, \phi(0))
$$

is valid whenever $\mathrm{V}(\mathrm{t}+\Theta, \phi(\Theta)) \leqslant \mathrm{V}(\mathrm{t}, \phi(0))$ for $-\mathrm{r} \leqslant \Theta \leqslant 0$ where $\Delta \subset \mathbb{R}^{\mathrm{n}}, 0 \in \Delta$. Then $\mathrm{V}\left(\mathrm{t}, \mathrm{x}\left(\mathrm{t} ; \mathrm{t}_{0}, \phi_{0}\right)\right) \leqslant$ $\max _{-r \leqslant \Theta \leqslant 0} V\left(t_{0}+\Theta, \phi_{0}(\Theta)\right) E_{q}\left(B\left(t-t_{0}\right)^{q}\right), t \in\left[t_{0}, \infty\right)$, where $x\left(t ; t_{0}, \phi_{0}\right)$ is the solution of (2.1) with initial data $\left(\mathrm{t}_{0}, \phi_{0}\right) \in \mathbb{R}_{+} \times \mathrm{C}[[-\mathrm{r}, 0], \Delta]$.

The claim of Lemma 2.8 is not true. We will give a counterexample.

Example 2.9. Consider the IVP for FrDDE (2.1) with $n=1, t_{0}=0, r=0$, and $f\left(t, x_{t}\right)=2 x(t), \psi(0)=x_{0}$. Then the solution of $(2.1)$ is $x(t)=x_{0} E_{q}\left(2 t^{q}\right)$.

Consider the Lyapunov function $V(t, x)=g(t) x^{2}$ where $g \in C\left([0, \infty), \mathbb{R}_{+}\right), g(0)=0$ is a nondecreasing function (for example $g(t)=\frac{t}{t+1}$ ). For any point $\phi(0) \in \mathbb{R}$ according to Case 2.1 in Example 2.5 we get $\mathrm{D}_{(2.1)}^{+} \mathrm{V}(\mathrm{t}, \phi(0))=2 \mathrm{~g}(\mathrm{t}) \phi(0) \mathrm{f}\left(\mathrm{t}, \phi_{0}\right)=4 \mathrm{~g}(\mathrm{t})(\phi(0))^{2}=4 \mathrm{~V}(\mathrm{t}, \phi(0))$. Then $\mathrm{V}(0, \phi(0))=\mathrm{g}(0)(\phi(0))^{2}=0$ and the inequality

$$
\mathrm{V}(\mathrm{t}, \mathrm{x}(\mathrm{t}))=\mathrm{g}(\mathrm{t}) \mathrm{x}_{0}^{2}\left(\mathrm{E}_{\mathrm{q}}\left(2 \mathrm{t}^{\mathrm{q}}\right)\right)^{2} \leqslant \mathrm{~V}(0, \phi(0)) \mathrm{E}_{\mathrm{q}}\left(4 \mathrm{t}^{\mathrm{q}}\right)=0
$$

is not true for $t \geqslant 0$. Therefore, the claim of Lemma 2.8 is not true in this particular case.

Remark 2.10. Note a similar result like in Lemma 2.8 is obtained in Theorem 4.4 [22] when the functional method is used instead of the Razumikhin method.

According to the above, the application of the derivative $D_{(2.1)}^{+} V(t, \phi(0))$ defined by (2.3) is not appropriate in studying stability properties of neural networks of fractional order (as presented in [25]). We will consider fractional neural networks with delays and using Lyapunov functions and their Caputo fractional derivative, Dini fractional derivative or Caputo fractional Dini derivative, we study stability.

\subsection{Stability results by Lyapunov functions for Caputo fractional differential equations.}

We will give some results for fractional derivatives of Lyapunov functions among Caputo fractional differential equations which will be used for our main results concerning the stability of neural networks. Recall the point $x^{*} \in \mathbb{R}^{n}$ is an equilibrium point of (2.1) iff $0=f\left(t, x^{*}\right), t>t_{0}$.

Definition 2.11. The equilibrium point $x^{*}$ of (2.1) is uniformly stable if for any $\varepsilon>0$ there exists $\delta>0$ such that any $\psi \in C\left([-r, 0], \mathbb{R}^{n}\right): \sup _{s \in[-r, 0]}\left\|\psi(s)-\chi^{*}\right\|<\delta$ implies $\left\|x(t)-x^{*}\right\|<\varepsilon$ for $t \geqslant t_{0}$ where $x(t)$ is a solution of the IVP for FrDDE (2.1) with the initial function $\psi$.

Lemma 2.12 ([8, Theorem 3.1]). Let $w_{1}, w_{2} \in C\left(\mathbb{R}_{+}, \mathbb{R}_{+}\right)$be nondecreasing functions, $w_{i}(s)>0, i=1,2$ for $s>0, w_{i}(0)=0, i=1,2, w_{2}(s)$ is strictly increasing, and there exists a continuously differentiable Lyapunov function $\mathrm{V}(\mathrm{t}, \mathrm{x}) \in \mathbb{R}_{+} \times \mathbb{R}^{\mathrm{n}} \rightarrow \mathbb{R}_{+}$such that

(i) $w_{1}(\|x\|) \leqslant V(t, x) \leqslant w_{2}(\|x\|), \quad t \geqslant 0, x \in \mathbb{R}^{n}$;

(ii) for any $\mathrm{t}>0$ such that $\sup _{\Theta \in[-r, t]} \mathrm{V}(\Theta, x(\Theta))=\mathrm{V}(\mathrm{t}, \mathrm{x}(\mathrm{t}))$, the inequality

$$
{ }_{0}^{C} D_{t}^{\beta} V(t, x(t)) \leqslant 0
$$

holds, where $\mathrm{x}(\mathrm{t})$ is a solution of (2.1) with $\mathrm{t}_{0}=0$.

Then the equilibrium point of (2.1) with $\mathrm{t}_{0}=0$ is uniformly stable. 
For the Caputo fractional Dini derivative we have the following.

Lemma 2.13 ([2, Theorem 6]). Assume $x^{*} \in \mathbb{R}^{n}$ is an equilibrium point for (2.1) with $\mathrm{T}=\infty$ and there exists a Lyapunov function $\mathrm{V}(\mathrm{t}, \mathrm{x}): \mathrm{V}(\mathrm{t}, 0)=0, \mathrm{t} \geqslant 0$, such that

(i) $\alpha_{1}(\|x\|) \leqslant V(t, x) \leqslant \alpha_{2}(\|x\|)$ for $t \geqslant 0, x \in \Delta$, where $\alpha_{i} \in C([0, \infty),[0, \infty)), i=1,2$ are strictly increasing and $\alpha_{i}(0)=0$;

(ii) for any initial data $\left(\mathrm{t}_{0}, \psi\right) \in \mathbb{R}_{+} \times \mathrm{C}([-\mathrm{r}, 0], \Delta)$ and any function $\phi \in \mathrm{C}([-\mathrm{r}, 0], \Delta)$ such that if for a point $\mathrm{t} \geqslant \mathrm{t}_{0}$ we have $\mathrm{V}(\mathrm{t}+\Theta, \phi(\Theta)) \leqslant \mathrm{V}(\mathrm{t}, \phi(0))$ for $\Theta \in[-\mathrm{r}, 0)$, then the inequality

$$
\stackrel{c}{c}(2.1) D_{+}^{q} V\left(t, \phi(0) ; t_{0}, \psi\right) \leqslant 0
$$

holds.

Then the equilibrium point of (2.1) is uniformly stable.

\section{Stability for Caputo reaction-diffusion fractional neural network with time-varying delays.}

\subsection{System description.}

Consider the general model of Caputo reaction-diffusion fractional neural network with time-varying delays (RDFrDNN)

$$
\begin{aligned}
{ }_{0}^{C} D_{t}^{q} u_{i}(t, x)= & -c_{i}(t) u_{i}(t, x)+\sum_{j=1}^{m} \frac{\partial}{\partial x_{j}}\left(D_{i j} \frac{\partial u_{i}(t, x)}{\partial x_{j}}\right)+\sum_{j=1}^{n} a_{i j}(t) f_{j}\left(u_{j}(t, x)\right) \\
& +\sum_{j=1}^{n} b_{i j}(t) g_{j}\left(u_{j}\left(t-\tau_{j}(t), x\right)\right)+I_{i}(t) \text { for } t>0, \quad i=1,2, \ldots, n,
\end{aligned}
$$

where $q \in(0,1), n$ represents the number of units in the network, $u_{i}(t, x), i=1,2, \ldots, n$ is the state of the $i$-th unit at time $t$ in space $x$ of master system, $x=\left[x_{1}, x_{2}, \ldots, x_{m}\right]^{T}, c_{i}(t)>0, i=1,2, \ldots, n$ representing the reset rate of the $i$-th neuron is the self-feedback term, $a_{i j}(t), b_{i j}(t), i, j=1,2, \ldots, n$ correspond to the connection of the $i$-th neuron to the $j$-th neuron at times $t$ and $t-\tau_{j}(t)$ respectively, $f_{j}$ and $g_{j}$ denote the activation functions of the neurons at time $t$ and $t-\tau_{j}(t)$, respectively, $f(u)=$ $\left[f_{1}\left(u_{1}\right), f_{2}\left(u_{2}\right), \ldots, f_{n}\left(u_{n}\right)\right]^{\top}, g(u)=\left[g_{1}\left(u_{1}\right), g_{2}\left(u_{2}\right), \ldots, g_{n}\left(u_{n}\right)\right]^{\top}$ and $I=\left[I_{1}, I_{2}, \ldots, I_{n}\right]^{\top}$ is an external bias vector, $\tau_{j}(t) \in C\left(\mathbb{R}_{+},[0, r]\right), j=1,2, \ldots, n$, are the transmission delays, and the smooth functions $D_{i j}=D_{i j}(t, x) \geqslant 0$ are the diffusion operators along the $i$-th neuron.

Further we will assume that

Assumption 3.1. The diffusion operators along the $i$-th neuron $D_{i j}=D_{i j}(t, x) \geqslant 0$ are smooth functions and there exist constants $d_{i j} \geqslant 0$ such that $D_{i j}(t, x) \geqslant d_{i j}$ for $t \geqslant 0, x \in \Delta$.

The initial value and boundary value conditions associated with the neural network (3.1) are listed as

$$
\begin{aligned}
& u_{i}(s, x)=\psi_{i}(s, x) \text { for }(s, x) \in[-r, 0] \times \Delta, \quad i=1,2, \ldots, n, \\
& u_{i}(t, x)=0 \text { for }(t, x) \in[-r, \infty) \times \partial \Delta, \quad i=1,2, \ldots, n,
\end{aligned}
$$

where $\psi_{i} \in C([-r, 0] \times \Delta, \mathbb{R}), i=1,2, \ldots, n$.

Let $\mathcal{C}=\mathrm{C}\left([-r, 0] \times \Delta, \mathbb{R}^{n}\right)$ and for $\psi \in \mathcal{C}, \psi=\left(\psi_{1}, \psi_{2}, \ldots, \psi_{n}\right)$ we define a norm

$$
\|\psi\|_{e}=\max _{s \in[-r, 0]} \sum_{i=1}^{n}\left[\int_{\Delta} \psi_{i}^{2}(s, x) d x\right]^{\frac{1}{2}} .
$$

Let $u \in C\left([0, \infty) \times \Delta, \mathbb{R}^{n}\right), u=\left(u_{1}, u_{2}, \ldots, u_{n}\right)$. For any $t \geqslant 0$ we define

$$
\left\|u_{i}(t, .)\right\|_{\Delta}=\left[\int_{\Delta} u_{i}^{2}(t, x) d x\right]^{\frac{1}{2}}, \quad\|u(t, .)\|_{\Delta}=\sum_{i=1}^{n}\left\|u_{i}(t, .)\right\|_{\Delta} .
$$

Also, denote by $|$.$| the absolute value of a scalar, and \|$.$\| is a norm in \mathbb{R}^{n}$. 
Remark 3.2. Reaction-diffusion neural network with time-varying delays of integer order are studied in $[16,17]$.

Remark 3.3. The problem of existence and uniqueness of equilibrium of fractional-order neural networks is investigated by several authors (see, for example, [7] for constant delays).

Definition 3.4. A vector $u^{*} \in \mathbb{R}^{n}, u^{*}=\left(u_{1}^{*}, u_{2}^{*}, \ldots, u_{n}^{*}\right)$ is an equilibrium point of RDFrDNN (3.1), iff the equalities $0=-c_{i}(t) u_{i}^{*}+\sum_{j=1}^{n} a_{i j}(t) f_{j}\left(u_{j}^{*}\right)+\sum_{j=1}^{n} b_{i j}(t) g_{j}\left(u_{j}^{*}\right)+I_{i}(t)$ hold for all $t>0, i=1,2, \ldots, n$.

We will discuss the equilibrium points on some RDFrDNN-s with various activation functions. It will be useful for further stability analysis.

Example 3.5. Let $n=m=1, c$ is a constant, $I_{\mathfrak{i}}(t) \equiv I$ and consider the scalar equation which is a special case of RDFrDNN (3.1):

$$
{ }_{0}^{C} D_{t}^{q} u(t, x)=-c u_{i}(t, x)+\frac{\partial}{\partial x}\left(D \frac{\partial u(t, x)}{\partial x}\right)+a(t) f(u(t, x))+b(t) g(u(t-\tau(t), x))+I
$$

Case 1. Let $I=0.5 \pi c$ and the activation function be the cosine function $f(u)=g(u)=\cos (u)$ (see [21]).

The point $u^{*}=0.5 \pi$ is an equilibrium point of RDFrDNN (3.3) because for all $t>0$ the equality $-0.5 \pi \mathrm{c}+\mathrm{a}(\mathrm{t}) \cos (0.5 \pi)+\mathrm{b}(\mathrm{t}) \cos (0.5 \pi)+0.5 \pi \mathrm{c}=0$ holds.

Case 2. Let $I=0.5 c$ and the activation function be the Probit function $f(u)=g(u)=\sqrt{2} \operatorname{erf}^{-1}(2 u-1)$ (see Figure 2) where $\operatorname{erf}(u)=\frac{2}{\sqrt{\pi}} \int_{0}^{u} e^{-t^{2}} d t$ is the error function. Then $\operatorname{erf}^{-1}(0)=0$.

The point $u^{*}=0.5$ is an equilibrium point of RDFrDNN (3.3) because $-c 0.5+a(t) f(0.5)+b(t) g(0.5)+$ $0.5 c=0$ for all $t>0$.

Case 3. Let $I=0.5 c$ and the activation function be the Logit function $f(u)=g(u)=\log \left(\frac{u}{1-u}\right)$ (see Figure 3). Then $f(0.5)=0$.

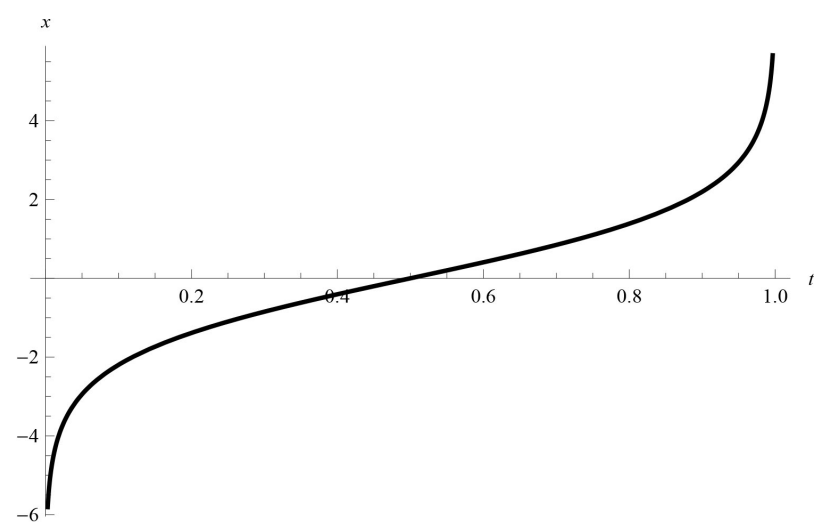

Figure 3: Example 3.5. Graph of the function $f(t)=$ $\log \left(\frac{t}{1-t}\right)$.

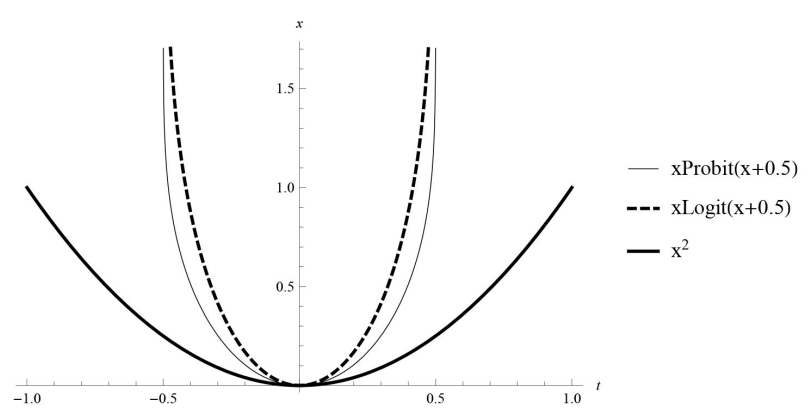

Figure 4: Example 3.21. Graph of the functions $x f(x+0.5)=x \sqrt{2} \operatorname{erf}^{-1}(2(x+0.5)-1), x f(x+0.5)=$ $x \log \left(\frac{x+0.5}{1-(x+0.5)}\right)$ and $x^{2}, x \in \mathbb{R}$.

The point $u^{*}=0.5$ is an equilibrium point of RDFrDNN (3.3) because $-c 0.5+a(t) f(0.5)+b(t) g(0.5)+$ $0.5 c=0$ for all $t>0$.

Consider the following assumption.

Assumption 3.6. Let the RDFrDNN (3.1) have an equilibrium point $u^{*} \in \mathbb{R}^{n}$.

If Assumption 3.6 is satisfied then we can shift the equilibrium point $u^{*}$ of system (3.1) to the origin. 
The transformation $y(t, x)=u(t, x)-u^{*}$ is used to put system (3.1) in the following form:

$$
\begin{aligned}
{ }_{0}^{C} D_{t}^{q} y_{i}(t, x)= & -c_{i}(t) y_{i}(t, x)+\sum_{j=1}^{m} \frac{\partial}{\partial x_{j}}\left(D_{i j} \frac{\partial y_{i}(t, x)}{\partial x_{j}}\right)+\sum_{j=1}^{n} a_{i j}(t) F_{j}\left(y_{j}(t, x)\right) \\
& +\sum_{j=1}^{n} b_{i j}(t) G_{j}\left(y_{j}\left(t-\tau_{j}(t), x\right)\right) \text { for } t>0, x \in \Delta, \quad i=1,2, \ldots, m,
\end{aligned}
$$

where $F_{j}(v)=f_{j}\left(v+u_{j}^{*}\right)-f_{j}\left(u_{j}^{*}\right), G_{j}(v)=g_{j}\left(v+u_{j}^{*}\right)-g_{j}\left(u_{j}^{*}\right), v \in \mathbb{R}, j=1,2, \ldots, n$.

\subsection{Stability analysis}

We will study stability properties of several different types of RDFrDNN (3.1) using different types of Lyapunov functions and their fractional derivatives given in Section 2.

Following the definitions given in Sections 2.1 we will use different types of fractional derivatives of Lyapunov functions among RDFrDNN (3.1) and its equivalent (3.4). Also, considering case 2.1 in Example 2.5 as well as Example 2.9 we will not use the definition given by Eq. (2.3).

For any $\mathrm{t} \geqslant 0, x \in \Delta, \mathrm{u} \in \mathrm{C}\left(\mathbb{R}_{+} \times \Delta, \mathbb{R}^{\mathrm{n}}\right), \mathrm{u}=\left(\mathrm{u}_{1}, \mathrm{u}_{2}, \ldots, \mathrm{u}_{\mathrm{n}}\right)$, and $v \in \mathrm{C}\left([-\mathrm{r}, \infty) \times \Delta, \mathbb{R}^{\mathrm{n}}\right), v=$ $\left(v_{1}, v_{2}, \ldots, v_{n}\right)$ we introduce the notation

$$
\begin{aligned}
\mathcal{F}(t, x, u, v) & =\left(\mathcal{F}_{1}(t, x, u, v), \mathcal{F}_{2}(t, x, u, v), \ldots, \mathcal{F}_{n}(t, x, u, v)\right): \\
\mathcal{F}_{i}(t, x, u, v) & =-c_{i}(t) u_{i}+\sum_{j=1}^{m} \frac{\partial}{\partial x_{j}}\left(D_{i j} \frac{\partial u_{i}}{\partial x_{j}}\right)+\sum_{j=1}^{n} a_{i j}(t) f_{j}\left(u_{j}\right)+\sum_{j=1}^{n} b_{i j}(t) g_{j}\left(v_{j}\right)+I_{i}(t) .
\end{aligned}
$$

\section{Example 3.7.}

Case 1. Let $\mathrm{V}(\mathrm{t}, \mathrm{x})=\sum_{i=1}^{n} x_{i}^{2}$. Let $\mathrm{u}(\mathrm{t}, \mathrm{x})$ be a solution of IVP for RDFrDNN (3.1), (3.2), and let $\mathrm{u}_{i}(\mathrm{t})=$ $\left\|u_{i}(t, .)\right\|_{\Delta} \in \mathrm{C}\left(\mathbb{R}_{+}, \mathbb{R}^{n}\right)$. Applying Lemma 1.2 we have

$$
\begin{aligned}
{ }_{0}^{C} D_{t}^{q} V(t, u(t))={ }_{0}^{C} D_{t}^{q} \sum_{i=1}^{n}\left\|u_{i}(t, .)\right\|_{\Delta}^{2} & =\sum_{i=1}^{n} \int_{\Delta}{ }_{0}^{C} D_{t}^{q} u_{i}^{2}(t, x) d x \leqslant 2 \sum_{i=1}^{n} \int_{\Delta} u_{i}(t, x){ }_{0}^{c} D_{t}^{q} u_{i}(t, x) d x \\
& =2 \sum_{i=1}^{n} \int_{\Delta} u_{i}(t, x) \mathcal{F}_{i}(t, x, u(t, x), u(t-\tau(t), x)) d x,
\end{aligned}
$$

where $u(t-\tau(t), x)=\left(u_{1}\left(t-\tau_{1}(t), x\right), u_{2}\left(t-\tau_{2}(t), x\right), \ldots, u_{n}\left(t-\tau_{n}(t), x\right)\right)$.

Case 2. Let $V(t, x)=p(t) \sum_{i=1}^{n} x_{i}^{2}$ where $p \in C\left([-r, \infty), \mathbb{R}_{+}: \alpha \leqslant p(t) \leqslant \beta\right.$ for $t \geqslant-r$ where $\alpha, \beta>0$ are constants, $x=\left(x_{1}, x_{2}, \ldots, x_{n}\right)$.

Let $\phi \in \mathrm{C}\left([-r, 0] \times \Delta, \mathbb{R}^{n}\right)$ and let $\Phi_{\mathfrak{i}}(\mathrm{t})=\left\|\phi_{\mathfrak{i}}(\mathrm{t}, .)\right\|_{\Delta} \in \mathrm{C}([-\mathrm{r}, 0], \mathbb{R}), \Phi=\left(\Phi_{1}, \Phi_{2}, \ldots, \Phi_{n}\right)$. Let the point $t>0: \sup _{\Theta \in[t-r, t]} V(t+\Theta, \Phi(\Theta))=\sup _{\Theta \in[t-r, t]} p(t+\Theta) \sum_{j=1}^{n}\left\|\phi_{j}(\Theta, .)\right\|_{\Delta}^{2}=p(t) \sum_{j=1}^{n}\left\|\phi_{j}(0, .)\right\|_{\Delta}^{2}$ $=\mathrm{V}(\mathrm{t}, \Phi(0))$. Then from formula (2.4) we obtain for the Dini fractional derivative:

$$
\begin{aligned}
& \mathcal{D}_{(2.1)}^{+} \mathrm{V}(\mathrm{t}, \Phi(0) ; 0)=\limsup _{\mathrm{h} \rightarrow 0} \frac{1}{\mathrm{~h}^{\mathrm{q}}}\left[\mathrm{p}(\mathrm{t}) \sum_{i=1}^{\mathrm{n}} \int_{\Delta} \phi_{i}^{2}(0, x) \mathrm{dx}\right. \\
& \left.-\sum_{r=1}^{\left[\frac{\mathrm{t}}{\mathrm{h}}\right]}(-1)^{\mathrm{r}+1}{ }_{\mathrm{q}} \mathrm{C}_{\mathrm{r}} \mathrm{p}(\mathrm{t}-\mathrm{rh}) \sum_{i=1}^{n} \int_{\Delta}\left(\phi_{i}(0, x)-\mathrm{h}^{\mathrm{q}} \mathcal{F}_{\mathfrak{i}}\left(\mathrm{t}, x, \phi_{i}(0, x), \phi_{i}\left(-\tau_{i}(0), x\right)\right)\right)^{2} \mathrm{~d} x\right] \\
& =2 p(t) \sum_{i=1}^{n} \int_{\Delta} \phi_{i}(0, x) \mathcal{F}\left(t, x, \phi_{i}(0, x), \phi_{i}\left(-\tau_{i}(t), x\right)\right) d x+\left(\sum_{i=1}^{n}\left\|\phi_{i}(0, .)\right\|_{\Delta}^{2}\right){ }_{0}^{R L} D^{q} p(t) .
\end{aligned}
$$

Definition 3.8. The equilibrium point $u^{*}$ of RDFrDNN (3.1) is uniformly stable if for any $\varepsilon>0$ there exists $\delta>0$ such that any $t_{0} \geqslant 0$ and $\psi \in \mathcal{C}:\left\|\psi-x^{*}\right\|_{\mathcal{e}}<\delta$ implies $\left\|u(t, .)-x^{*}\right\|_{\Delta}<\varepsilon$ for $t \geqslant t_{0}$, where $u(t, x)$ is a solution of the IVP for RDFrDNN (3.1), (3.4). 


\subsubsection{Lipschitz activation functions and quadratic Lyapunov functions}

Stability analysis of the fractional-order neural networks with constant delays and Lipschitz active functions was studied in [12] and the argument was based on topological degree theory, nonsmooth analysis and a nonlinear measure method. We will apply the Lyapunov method to derive some sufficient conditions for stability in the case of variable delays.

The reaction-diffusion cellular neural networks of fractional-order with delays is also studied in [25] in the case of Lipschitz activation functions. Unfortunately there are several inaccuracies in [25]:

- the domain of the defined function F in Definition 6 [25] contradicts its application in the Caputo fractional differential equation;

- the proofs of the main Theorems 11 and 16 [25] are based on the application of Lemma 2.8 whose claim is not true (see Example 2.9);

- the incorrect inequality $\sum_{j=1}^{m} \max _{s \in[-r, 0]}\left\|\tilde{\varphi}_{i}(s, .)\right\|^{2} \leqslant \max _{s \in[-r, 0]} \sum_{j=1}^{m}\left\|\tilde{\varphi}_{i}(s, .)\right\|^{2}$ is used in the proof of Theorems 11 and 16 in [25].

We will state the result in the case of Lipschitz activation functions and variable bounded coefficients. The case of multiple time constant delays and constant functions of the connection of the $i$-th neuron to the $j$-th neuron in RDFrDNN (3.1) is studied using the quadratic Lyapunov function in [33].

We will assume the following.

Assumption 3.9. The neuron activation functions are Lipschitz, i.e., there exist positive numbers $L_{i}, H_{i}$, $i=1,2, \ldots, n$ such that $\left|f_{i}(u)-f_{i}(v)\right| \leqslant L_{i}|u-v|$ and $\left|g_{i}(u)-g_{i}(v)\right| \leqslant H_{i}|u-v|, i=1,2, \ldots, n$ for $u, v \in \mathbb{R}$.

Assumption 3.10. There exists positive numbers $M_{i, j}, C_{i, j}, i, j=1,2, \ldots, n$ such that $\left|a_{i, j}(t)\right| \leqslant M_{i, j}$, $\left|b_{i, j}(t)\right| \leqslant C_{i, j}$ for $t>0$.

Assumption 3.11. The inequality

$$
2\left(\min _{i=1, n} c_{i}+\min _{i=\overline{1, n}} \sum_{j=1}^{m} \tilde{d}_{i j}\right)>\sum_{i=1}^{n}\left(\max _{j=\overline{1, n}} M_{i j} L_{j}+\max _{j=\overline{1, n}} C_{i j} H_{j}\right)+\max _{i=1, n}\left(\sum_{j=1}^{n}\left(M_{i j} L_{j}+C_{i j} H_{j}\right)\right.
$$

holds.

Remark 3.12. If Assumption 3.9 is satisfied then the functions $F, G$ in $\operatorname{RDFrDNN}(3.1)$ satisfy $\left|F_{j}(u)\right| \leqslant$ $L_{j}|u|,\left|G_{j}(u)\right| \leqslant H_{j}|u|, j=1,2, \ldots, n$ for any $u \in \mathbb{R}$.

Theorem 3.13. Let $\Delta=\left\{x \in \mathbb{R}^{m}:\left|x_{i}\right| \leqslant l_{i}, i=1,2, \ldots, m\right\}, l_{i}, i=1,2, \ldots, m$ are positive constants, $c_{j}(t) \geqslant c_{j}>0, j=1,2, \ldots, n$, and ssumptions $3.1,3.6,3.9,3.10$, and 3.11 are satisfied with $\tilde{d}_{i j}=\frac{d_{i j}}{l_{j}^{2}}$. Then the equilibrium point $x^{*}$ of RDFrDNN (3.1) is uniformly stable.

Proof. Consider the quadratic functions $\mathrm{V}(\mathrm{t}, \mathrm{u})=\sum_{i=1}^{n} u_{i}^{2}, u \in \mathbb{R}^{n}$. Let $u(t, x)$ be a solution of IVP for $\operatorname{RDFrDNN}$ (3.1), (3.2) and let $\mathrm{u}_{i}(\mathrm{t})=\left\|\mathrm{u}_{\mathrm{i}}(\mathrm{t}, .)\right\|_{\Delta} \in \mathrm{C}\left(\mathbb{R}_{+}, \mathbb{R}^{n}\right)$. Let the point $\mathrm{t}>0$ be such that $\sup _{\Theta \in[-r, t]} \mathrm{V}(\Theta, \mathrm{U}(\Theta))=\sup _{\Theta \in[-r, t]} \sum_{j=1}^{n}\left\|u_{j}(\Theta, .)\right\|_{\Delta}^{2}=\sum_{j=1}^{n}\left\|u_{j}(t, .)\right\|_{\Delta}^{2}=V(t, u(t))$. Then since $\tau_{i}(t) \in$ $[0, r]$ we have $\sum_{j=1}^{n}\left\|u_{j}(t, .)\right\|_{\Delta}^{2} \geqslant \sum_{j=1}^{n}\left\|u_{j}\left(t-\tau_{j}(t), .\right)\right\|_{\Delta}^{2}, t \geqslant 0$. According to Eq. (2.7) we get

$$
\begin{aligned}
{ }_{0}^{C} D_{t}^{q} V(t, u(t)) \leqslant & 2 \sum_{i=1}^{n} \int_{\Delta} u_{i}(t, x) \mathcal{F}_{i}(t, x, u(t, x), u(t-\tau(t), x)) d x \\
\leqslant & -2 \sum_{i=1}^{n} c_{i} \int_{\Delta} u_{i}^{2}(t, x) d x+2 \sum_{i=1}^{n} \sum_{j=1}^{m} \int_{\Delta} \frac{\partial}{\partial x_{j}}\left(D_{i j} \frac{\partial u_{i}(t, x)}{\partial x_{j}}\right) u_{i}(t, x) d x \\
& +2 \sum_{i=1}^{n} \sum_{j=1}^{n}\left|a_{i j}(t)\right| \int_{\Delta}\left|F_{j}\left(u_{j}(t, x)\right)\right|\left|u_{i}(t, x)\right| d x
\end{aligned}
$$




$$
\begin{aligned}
& +2 \sum_{i=1}^{n} \sum_{j=1}^{n}\left|b_{i j}(t)\right| \int_{\Delta}\left|G_{j}\left(u_{j}(t-\tau(t), x)\right)\right|\left|u_{i}(t, x)\right| d x \\
& \leqslant-2 \sum_{i=1}^{n} c_{i} \int_{\Delta} u_{i}^{2}(t, x) d x-2 \sum_{i=1}^{n} \sum_{j=1}^{m} \int_{\Delta} \frac{d_{i j}}{l_{j}^{2}} u_{i}^{2}(t, x) d x \\
& +2 \sum_{i=1}^{n} \sum_{j=1}^{n} M_{i j} \int_{\Delta} L_{j}\left|u_{j}(t, x) \| u_{i}(t, x)\right| d x \\
& +2 \sum_{i=1}^{n} \sum_{j=1}^{n} C_{i j} \int_{\Delta} H_{j}\left|u_{j}(t-\tau(t), x) \| u_{i}(t, x)\right| d x \\
& \leqslant-2\left(\min _{i} c_{i}+\min _{i} \sum_{j=1}^{m} \frac{d_{i j}}{l_{j}^{2}}\right)\|u(t, .)\|_{\Delta}^{2}+\left(\sum_{i=1}^{n}\left(\max _{j} M_{i j} L_{j}+\max _{j} C_{i j} H_{j}\right)\right)\|u(t, .)\|_{\Delta}^{2} \\
& +\left(\max _{i}\left(\sum_{j=1}^{n}\left(M_{i j} L_{j}+C_{i j} H_{j}\right)\right)\|u(t, .)\|_{\Delta}^{2} \leqslant 0,\right.
\end{aligned}
$$

where $u(t-\tau(t), x)=\left(u_{1}\left(t-\tau_{1}(t), x\right), u_{2}\left(t-\tau_{2}(t), x\right), \ldots, u_{n}\left(t-\tau_{n}(t), x\right)\right)$.

The above inequality and Lemma 2.12 prove the claim.

Remark 3.14. Note in this case the sufficient conditions for stability do not depend on the order q of the Caputo fractional derivative.

Example 3.15. Consider the system of RDFrDNN (3.1) with $n=3, m=2, c_{i}(t) \equiv c_{i}, i=1,2,3, l_{i}=$ $2, i=1,2$, with the activation functions $f_{j}(s)=g_{j}(s)=0.5 \tanh (s), j=1,2,3$, the delay $\tau(t) \equiv 1$ and $\left|a_{i j}(t)\right| \leqslant M_{i j},\left|b_{i j}(t)\right| \leqslant C_{i j}, i, j=1,2,3, t \geqslant 0$, where $M=\left\{M_{i j}\right\}, C=\left\{C_{i j}\right\}$ are given by

$$
M=\left(\begin{array}{ccc}
-0.1 & 0.5 & 0.3 \\
-0.2 & 0.3 & 0.2 \\
0.4 & -0.2 & -0.1
\end{array}\right), \quad C=\left(\begin{array}{ccc}
0.1 & -0.1 & -0.2 \\
0.3 & 0.2 & -0.1 \\
-0.2 & 0.5 & 0.3
\end{array}\right)
$$

and

$$
\left(D_{i j}\right)_{3 \times 2}=\left(\begin{array}{cc}
3+\sin t & 0 \\
\sin ^{2} t & \cos ^{2} t \\
0 & 4+\cos t
\end{array}\right), \quad\left(d_{i j}\right)_{3 \times 2}=\left(\begin{array}{ll}
2 & 0 \\
0 & 0 \\
0 & 3
\end{array}\right) .
$$

The point $x^{*}=(0,0,0)$ is an equilibrium point of Caputo FODNN (3.1) if $I_{i}(t) \equiv 0, i=1,2,3$ (see Example 3.5).

Then $L_{i}=H_{i}=0.5$ and $\sum_{i=1}^{3}\left(\max _{j=1,3} M_{i j} L_{j}+\max _{j=1,3} C_{i j} H_{j}\right)=(0.25+0.1)+(0.15+0.15)+(0.2+$ $0.25)=1.1, \max _{i=1,3}\left(\sum_{j=1}^{3}\left(M_{i j} L_{j}+C_{i j} H_{j}\right)=0.85, \min _{i=1,3} \sum_{j=1}^{2} \tilde{d}_{i j}=0.25\right.$.

Therefore, if $c_{i}>\frac{1.95}{2}-0.25=0.725, i=1,2,3$ then according to Theorem 3.13 the zero equilibrium is uniformly stable.

\subsubsection{Non-Lipschitz activation functions and quadratic Lyapunov functions}

There are many types of activation functions which are not Lipschitz (see Example 3.5, Cases 2 and 3). In this case we assume:

Assumption 3.16. There exists a function $\xi \in C\left(\mathbb{R}_{+}, \mathbb{R}\right)$ such that for any solution $u(t, x)$ of $\operatorname{RDFrDNN}$ (3.1) and any point $t>0$ such that $\sup _{\Theta \in[-r, t]} \sum_{j=1}^{n}\left\|u_{j}(\Theta, .)\right\|_{\Delta}^{2}=\sum_{j=1}^{n}\left\|u_{j}(t, .)\right\|_{\Delta}^{2}$ the inequalities

$$
\begin{aligned}
& \sum_{j=1}^{n} a_{i j}(t) \int_{\Delta}\left(f_{j}\left(u_{j}(t, x)+u_{j}^{*}\right)-f_{j}\left(u_{j}^{*}\right)\right) u_{i}(t, x) d x \\
& \quad+\sum_{j=1}^{n} b_{i j}(t) \int_{\Delta}\left(g_{j}\left(u_{j}\left(t-\tau_{j}(t), x\right)+u_{j}^{*}\right)-g_{j}\left(u_{j}^{*}\right)\right) u_{i}(t, x) d x \leqslant \xi(t) \int_{\Delta} u_{i}^{2}(t, x) d x \text { for } i=1,2, \ldots, n
\end{aligned}
$$

hold. 
Assumption 3.17. There exists a function $\eta \in C\left(\mathbb{R}_{+},(0, \infty)\right)$ such that the inequalities $c_{\mathfrak{i}}(t) \geqslant \eta(t), i=$ $1,2, \ldots, n, t \geqslant 0$ hold.

Remark 3.18. Note that, if the functions $f_{j}, g_{j}, j=1,2, \ldots, n$ are Lipschitz then condition 3.16 is satisfied.

Theorem 3.19. Let $\Delta=\left\{x \in \mathbb{R}^{m}:\left|x_{i}\right| \leqslant l_{i}, i=1,2, \ldots, m\right\}$, and Assumptions 3.1, 3.6, 3.16, and 3.17 be satisfied with $\xi(t) \leqslant \eta(t)+\min _{i=\overline{1, n}} \sum_{j=1}^{m} \frac{d_{i j}}{l_{j}^{2}}, t \geqslant 0$. Then the equilibrium point $u^{*}$ of $R D F r D N N$ (3.1) is uniformly stable.

Proof. Consider the quadratic functions $V(t, u)=\sum_{i=1}^{n} u_{i}^{2}, u \in \mathbb{R}^{n}$. Let $u(t, x)$ be a solution of IVP for $\operatorname{RDFrDNN}(3.1)$ and let $\mathrm{u}_{i}(\mathrm{t})=\left\|\mathrm{u}_{i}(\mathrm{t}, .)\right\|_{\Delta} \in \mathrm{C}\left(\mathbb{R}_{+}, \mathbb{R}\right)$. Let the point $\mathrm{t}>0$ be such that

$$
\sup _{\Theta \in[-r, t]} V(\Theta, u(\Theta))=\sup _{\Theta \in[-r, t]} \sum_{j=1}^{n}\left\|u_{j}(\Theta, .)\right\|_{\Delta}^{2}=\sum_{j=1}^{n}\left\|u_{j}(t, .)\right\|_{\Delta}^{2}=V(t, u(t)) .
$$

Then since $\tau_{j}(t) \in[0, r], j=1,2, \ldots, n$, we have

$$
\sum_{j=1}^{n}\left\|u_{j}(t, .)\right\|_{\Delta}^{2} \geqslant \sum_{j=1}^{n}\left\|u_{j}\left(t-\tau_{j}(t), .\right)\right\|_{\Delta}^{2}, t \geqslant 0 .
$$

According to Eq. (2.7), Assumptions 3.16 and 3.17, and Lemma 1.2 we get for the Caputo fractional derivative

$$
\begin{aligned}
{ }_{0}^{C} D_{t}^{q} V(t, u(t)) \leqslant & 2 \sum_{i=1}^{n} \int_{\Delta} u_{i}(t, x) \mathcal{F}_{i}(t, x, u(t, x), u(t-\tau(t), x)) d x \\
= & -2 \sum_{i=1}^{n} c_{i} \int_{\Delta} u_{i}^{2}(t, x) d x+2 \sum_{i=1}^{n} \sum_{j=1}^{m} \int_{\Delta} \frac{\partial}{\partial x_{j}}\left(D_{i j} \frac{\partial u_{i}(t, x)}{\partial x_{j}}\right) u_{i}(t, x) d x \\
& +2 \sum_{i=1}^{n} \sum_{j=1}^{n} a_{i j}(t) \int_{\Delta} F_{j}\left(u_{j}(t, x)\right) u_{i}(t, x) d x \\
& +2 \sum_{i=1}^{n} \sum_{j=1}^{n} b_{i j}(t) \int_{\Delta} G_{j}\left(u_{j}(t-\tau(t), x)\right) u_{i}(t, x) d x \\
\leqslant & -2\left(\eta(t)+\min _{i=1, n} \sum_{j=1}^{m} \frac{d_{i j}}{l_{j}^{2}}-\xi(t)\right) \sum_{i=1}^{n}\left\|u_{i}(t, .)\right\|_{\Delta}^{2},
\end{aligned}
$$

where $u(t-\tau(t), x)=\left(u_{1}\left(t-\tau_{1}(t), x\right), u_{2}\left(t-\tau_{2}(t), x\right), \ldots, u_{n}\left(t-\tau_{n}(t), x\right)\right)$.

From inequality (3.5) and Lemma 2.12 the claim follows.

Remark 3.20. Note that, in this case the sufficient conditions for stability depend indirectly on the order $\mathrm{q}$ of the Caputo fractional derivative.

Example 3.21. Let $n=1, m=1, l=1$ and consider the scalar nonlinear RDFrDNN for $t>0$

$$
{ }_{0}^{C} D_{t}^{q} u(t, x)=-c(t) u(t, x)+\frac{\partial}{\partial x}\left(D \frac{\partial u(t, x)}{\partial x}\right)+a(t) f(u(t, x))+b(t) f(u(t-\tau(t), x))+I(t),
$$

where $q \in(0,1), c \in C([0, \infty),(0, \infty)), I=0.5 c(t), D \geqslant 0, \tau \in C\left(\mathbb{R}_{+},[0, r]\right), r=$ const $>0, \Delta=\{x \in \mathbb{R}:$ $|x| \leqslant l\}$, the functions $a \in C([0, \infty),(\infty, 0]), b \in C([0, \infty),[0, \infty))$ are such that $a(t)+b(t)=\mu(t) \leqslant 0$ and the activation function $f(t)$ is the Probit function or the Logit function. Then the equation (3.6) has an equilibrium point $x^{*}=0.5$ (see Cases 2 and 3 in Example 3.5). Both activation functions are not Lipschitz and Theorem 3.13 cannot be applied. 
Let $u(t, x)$ be a solution of the scalar nonlinear RDFrDNN (3.6) and $t>0$ be such that

$$
\sup _{\Theta \in[-r, t]}\|\mathfrak{u}(\Theta, .)\|_{\Delta}^{2}=\|\mathfrak{u}(t, .)\|_{\Delta}^{2} \quad \text { or } \sup _{\Theta \in[-r, t]}|u(\Theta, x)|=|\mathfrak{u}(t, x)|, x \in \Delta .
$$

Consider the following possible cases:

Case 1. Let $u(t, x)<0$ and $u(t-\tau(t), x)<-0.5<0$. Since $\tau(t) \geqslant 0$ we obtain $u(t-\tau(t), x) \geqslant u(t, x)$ and from the monotonicity property of the function $f$ we get $f(u(t-\tau(t), x)+0.5) \geqslant f(u(t, x)+0.5)$ and $b(t) f(u(t-\tau(t), x)+0.5) u(t, x) \leqslant b(t) f(u(t, x)+0.5) u(t, x)$. Then applying $x f(x+0.5) \geqslant x^{2} \geqslant$ $0, x \in \mathbb{R}$ (see Figure 4 ) we get

$$
\begin{aligned}
& a(t) f(u(t, x)+0.5) u(t, x)+b(t) f(u(t-\tau(t), x)+0.5) u(t, x) \\
& \quad \leqslant(a(t)+b(t)) f(u(t, x)+0.5) u(t, x) \leqslant \mu(t) u^{2}(t, x) .
\end{aligned}
$$

Case 2. Let $u(t, x)<0$ and $u(t-\tau(t), x)>-0.5$. Then $f(u(t-\tau(t), x)+0.5) \geqslant 0$ and applying $x f(x+$ $0.5) \geqslant x^{2} \geqslant 0, x \in \mathbb{R}$ we obtain

$$
\begin{aligned}
& a(t) f(u(t, x)+0.5) u(t, x)+b(t) f(u(t-\tau(t), x)+0.5) u(t, x) \\
& \quad \leqslant a(t) f(u(t, x)+0.5) u(t, x) \leqslant a(t) u^{2}(t, x) \leqslant \mu(t) u^{2}(t, x) .
\end{aligned}
$$

Case 3. Let $u(t, x)>0$. Then $|u(t-\tau(t), x)| \leqslant u(t, x)$ and from the monotonicity property of the function $f$ it follows that $f(u(t-\tau(t), x)+0.5) \leqslant f(|u(t-\tau(t), x)|+0.5) \leqslant f(u(t, x)+0.5)$. Therefore,

$$
\begin{aligned}
& a(t) f(u(t, x)+0.5) u(t, x)+b(t) f(u(t-\tau(t), x)+0.5) u(t, x) \\
& \quad \leqslant(a(t)+b(t)) f(u(t, x)+0.5) u(t, x) \leqslant \mu(t) u^{2}(t, x) .
\end{aligned}
$$

Then the inequality

$$
\int_{\Delta}(a(t) f(u(t, x)+0.5) u(t, x)+b(t) f(u(t-\tau(t), x)+0.5) u(t, x)) d x \leqslant \mu(t) \int_{\Delta} u^{2}(t, x) d x, t>0
$$

holds. Therefore, Assumption 3.16 is satisfied.

Then according to Theorem 3.19, the equilibrium point $x^{*}=0.5$ of $\operatorname{RDFrDNN}(3.6)$ is uniformly stable for all $c(t)>0$.

\subsubsection{Non-Lipschitz activation functions and time-depended Lyapunov functions.}

In the case the function $\eta(t)$ in Assumption 3.17 is not large enough so we assume:

Assumption 3.22. There exists a continuous positive function $p(t) \in C\left([0, \infty), \mathbb{R}_{+}\right)$such that $0<\alpha \leqslant$ $p(t) \leqslant \beta$ and the fractional derivative ${ }_{0}^{R L} D_{t}^{q} p(t)$ exists for $t>0$.

In this case Assumption 3.16 could be weakened to

Assumption 3.23. There exists a function $\xi \in C\left(\mathbb{R}_{+}, \mathbb{R}\right)$ such that for any function $\phi \in C\left([-r, 0], \mathbb{R}^{n}\right)$ and $t>0$ be such that $\sup _{\Theta \in[-r, 0]} p(t+\Theta) \sum_{j=1}^{n}\left\|\phi_{j}(\Theta, .)\right\|_{\Delta}^{2}=p(t) \sum_{j=1}^{n}\left\|\phi_{j}(0, .)\right\|_{\Delta}^{2}$ and the inequality

$$
\begin{aligned}
& \sum_{i=1}^{n} \sum_{j=1}^{n} a_{i j}(t) \int_{\Delta}\left(f_{j}\left(\phi_{j}(0, x)+u_{j}^{*}\right)-f_{j}\left(u_{j}^{*}\right)\right) \phi_{i}(0, x) d x \\
& \quad+\sum_{j=1}^{n} b_{i j}(t) \int_{\Delta}\left(g_{j}\left(\phi_{j}\left(-\tau_{j}(0), x\right)+u_{j}^{*}\right)-g_{j}\left(u_{j}^{*}\right)\right) \phi_{i}(0, x) d x \leqslant \xi(t) \sum_{i=1}^{n}\left\|\phi_{i}(0, .)\right\|_{\Delta}^{2}
\end{aligned}
$$

holds. 
Theorem 3.24. Let $\Delta=\left\{x \in \mathbb{R}^{m}:\left|x_{i}\right| \leqslant l_{i}, i=1,2, \ldots, m\right\}, c_{j}(t) \equiv c_{j}>0, j=1,2, \ldots, n$ and Assumptions $3.1,3.6,3.17,3.22$, and 3.23 be satisfied and

$$
-p(t)\left(\eta(t)+\min _{i=1, n} \sum_{j=1}^{m} \frac{d_{i j}}{l_{j}^{2}}-\xi(t)\right)+0.50_{t}^{R L} D_{t}^{q} p(t) \leqslant 0, t>0 .
$$

Then the equilibrium point $\mathrm{u}^{*}$ of RDFrDNN (3.1) is uniformly stable.

Proof. In this case the quadratic function $V(t, u)=\sum_{i=1}^{n} u_{i}^{2}, u \in \mathbb{R}^{n}$ does not work.

Consider the Lyapunov function $V(t, u)=p(t) \sum_{i=1}^{n} u_{i}^{2}, u \in \mathbb{R}^{n}$, where the function $p(t)$ is defined in Assumption 3.22. Then according to Assumption 3.17, condition (i) of Lemma 2.13 is satisfied with $\alpha_{1}(u)=\alpha u$ and $\alpha_{2}(u)=\beta u$.

Let $\phi \in \mathrm{C}\left([-r, 0] \times \Delta, \mathbb{R}^{n}\right)$ and let $\Phi_{\mathfrak{i}}(\mathrm{t})=\left\|\phi_{\mathfrak{i}}(\mathrm{t}, .)\right\|_{\Delta} \in \mathrm{C}([-r, 0], \mathbb{R})$. Let the point $\mathrm{t}>0$ be such that $\sup _{\Theta \in[-r, 0]} \mathrm{V}(\mathrm{t}+\Theta, \Phi(\Theta))=\sup _{\Theta \in[-r, 0]} p(t+\Theta) \sum_{j=1}^{n}\left\|\phi_{j}(\Theta, .)\right\|_{\Delta}^{2}=p(t) \sum_{j=1}^{n}\left\|\phi_{j}(0, .)\right\|_{\Delta}^{2}=\mathrm{V}(\mathrm{t}, \Phi(0))$. Then from formula (2.4) and Eq. (3.5) we obtain for the Dini fractional derivative:

$$
\begin{aligned}
\mathcal{D}_{(3.4)}^{+} & V(t, \Phi(0) ; 0) \\
& =2 p(t) \sum_{i=1}^{n} \int_{\Delta} \phi_{i}(0, x) \mathcal{F}_{i}(t, x, \phi(0, x), \phi(-\tau(t), x)) d x+\left(\sum_{i=1}^{n}\left\|\phi_{i}(0, .)\right\|_{\Delta}^{2}\right){ }_{0}^{R L} D^{q} p(t) \\
& \leqslant-2 p(t)\left(\eta(t)+\min _{i=\overline{1, n}} \sum_{j=1}^{m} \frac{d_{i j}}{l_{j}^{2}}-\xi(t)\right) \sum_{i=1}^{n}\left\|\phi_{i}(0, .)\right\|_{\Delta}^{2}+\left(\sum_{i=1}^{n}\left\|\phi_{i}(0, .)\right\|_{\Delta}^{2}\right){ }_{0}^{R L} D^{q} p(t) \\
& \leqslant\left(-2 p(t) \eta(t)-2 p(t) \min _{i=1, n} \sum_{j=1}^{m} \frac{d_{i j}}{l_{j}^{2}}+2 p(t) \xi(t)+{ }_{0}^{R L} D_{t}^{q} p(t)\right) \sum_{i=1}^{n}\left\|\phi_{i}(0, .)\right\|_{\Delta}^{2},
\end{aligned}
$$

where $\phi(-\tau(t), x)=\left(\phi_{1}\left(-\tau_{1}(t), x\right), \phi_{2}\left(-\tau_{2}(t), x\right), \ldots, \phi_{n}\left(-\tau_{n}(t), x\right)\right)$.

According to Remark 2.3 and inequality (3.7) we get the inequality

$$
{ }_{(3.4)}^{c} \mathrm{D}_{+}^{\mathrm{q}} \mathrm{V}\left(\mathrm{t}, \Phi(0) ; 0, \psi_{0}\right)<\mathcal{D}_{(3.4)}^{+} \mathrm{V}(\mathrm{t}, \Phi(0) ; 0) \leqslant 0,
$$

i.e., condition (ii) of Lemma 2.13 is satisfied. Applying Lemma 2.13 we prove the claim.

Remark 3.25. Note that, in this case the sufficient conditions for stability depend on the order $q$ of the Caputo fractional derivative.

Example 3.26. Consider scalar RDFrDNN (3.1) with $\mathrm{n}=1, \mathrm{~m}=2, \mathrm{l}_{1}=\mathrm{l}_{2}=4, \mathrm{c}(\mathrm{t})=\frac{0.55}{\mathrm{t}^{\mathrm{q}} \Gamma(1-\mathrm{q})\left(\mathrm{E}_{\mathrm{q}}\left(-\mathrm{t}^{\mathrm{q}}\right)+0.1\right)}+$ $\frac{0.005}{\mathrm{E}_{\mathrm{q}}\left(-\mathrm{t}^{\mathrm{q}}\right)+0.1}, \mathrm{I}(\mathrm{t}) \equiv 0, \mathrm{a}, \mathrm{b} \in \mathrm{C}\left(\mathbb{R}_{+}, \mathbb{R}_{+}\right): \mathrm{a}(\mathrm{t})+\mathrm{b}(\mathrm{t})=\frac{0.45 \mathrm{E}_{\mathrm{q}}\left(-\mathrm{t}^{\mathrm{q}}\right)}{\mathrm{E}_{\mathbf{q}}\left(-\mathrm{t}^{\mathrm{q}}\right)+0.1}$, the activation functions are the Continuous Tan-Sigmoid Function $\mathrm{f}(\mathrm{u})=\mathrm{g}(\mathrm{u})=\tanh (\mathrm{u})=\frac{\mathrm{e}^{\mathrm{u}}-\mathrm{e}^{-u}}{e^{u}+e^{-u}}, \tau(\mathrm{t}) \equiv \mathrm{r}>0$, the equilibrium point $u^{*}=0,\left(D_{i j}\right)_{1 \times 2}=\left(\begin{array}{lll}0.2 & 0.7 & 0.1\end{array}\right)$, and $\Delta=\left\{x \in \mathbb{R}^{2}:\left|x_{i}\right| \leqslant 4, i=1,2\right\}$.

Theorem 3.13 is not applicable since the coefficient before $x$ is not bounded by a constant for $t \geqslant 0$.

Let the function $u(t, x)$ be a solution of the scalar RDFrDNN (3.1) and the point $t>0$ be such that $\sup _{\Theta \in[-r, 0]}\|u(t+\Theta, .)\|_{\Delta}^{2}=|| u(t,.) \|_{\Delta}^{2}$, i.e., $\sup _{\Theta \in[-r, 0]}|u(t+\Theta, x)|=|u(t, x)|$ for $x \in \Delta$.

Consider the following possible cases:

Case 1. Let $u(t, x)<0$ and $u(t-r, x)<0$. Then we obtain $u(t-r, x) \geqslant u(t, x)$ and from the monotonicity property of the function $f$ we get $f(u(t-r, x)) \geqslant f(u(t, x))$ and

$$
b(t) f(u(t-r, x)) u(t, x) \leqslant b(t) f(u(t, x)) u(t, x) .
$$

Then applying $|f(u(t, x))|=-f(u(t, x)) \leqslant|u(t, x)|=-u(t, x)$ (see Figure 5) and $b(t) \geqslant 0$ we get

$$
a(t) f(u(t, x)) u(t, x)+b(t) f(u(t-r, x)) u(t, x) \leqslant(a(t)+b(t)) u^{2}(t, x) .
$$


Case 2. Let $u(t, x)<0$ and $u(t-r, x)>0$. Then $f(u(t-r, x)) \geqslant 0$ and note $|f(u(t-r, x))|=f(u(t-r, x)) \geqslant$ $|u(t, x)|=-u(t, x) \geqslant 0$ and $f(u(t-r, x)) u(t, x) \leqslant-u^{2}(t, x) \leqslant 0$. Then we obtain

$a(t) f(u(t, x)) u(t, x)+b(t) f(u(t-r, x)) u(t, x) \leqslant a(t) f(u(t, x)) u(t, x) \leqslant a(t) u^{2}(t, x) \leqslant(a(t)+b(t)) u^{2}(t, x)$.

Case 3. Let $u(t, x)>0$. Then $|u(t-r, x)| \leqslant u(t, x)$ and from the monotonicity property of the function $f$ it follows that $f(u(t-r, x)) \leqslant f(|u(t-r, x)|) \leqslant f(u(t, x))$. Therefore,

$$
a(t) f(u(t, x)) u(t, x)+b(t) f(u(t-r, x)) u(t, x) \leqslant(a(t)+b(t)) f(u(t, x)) u(t, x) \leqslant(a(t)+b(t)) u^{2}(t, x) .
$$

Therefore, Assumption 3.17 is satisfied with $\xi(t) \equiv a(t)+b(t)$. However the inequality $\xi(t)=a(t)+$ $\mathrm{b}(\mathrm{t})=\frac{0.45 \mathrm{E}_{\mathrm{q}}\left(-\mathrm{t}^{\mathrm{q}}\right)}{\mathrm{E}_{\mathrm{q}}\left(-\mathrm{t}^{\mathrm{q}}\right)+0.1} \leqslant \eta(\mathrm{t})=\mathrm{c}(\mathrm{t})+\sum_{j=1}^{3} \frac{\mathrm{d}_{\mathrm{j}}}{l_{j}^{2}}=\frac{0.55}{\mathrm{t}^{\mathrm{q}} \Gamma(1-\mathrm{q})\left(\mathrm{E}_{\mathrm{q}}\left(-\mathrm{t}^{\mathrm{q}}\right)+0.1\right)}+\frac{0.005}{\mathrm{E}_{\mathrm{q}}\left(-\mathrm{t}^{\mathrm{q}}\right)+0.1}+\frac{1}{16}$ is not satisfied (see Figure 6 for $\mathrm{q}=0.8$ ). Therefore, Theorem 3.19 cannot be applied.

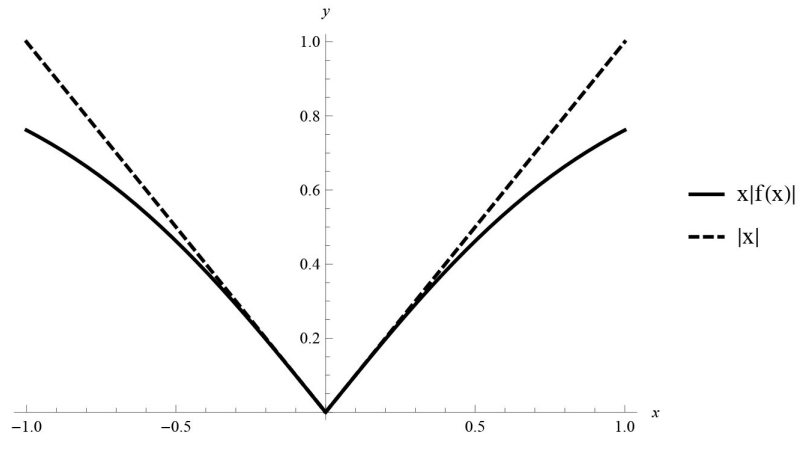

Figure 5: Example 3.26. Graph of the functions $|f(x)|=$ $|\tanh (x)|$ and $|x|$.

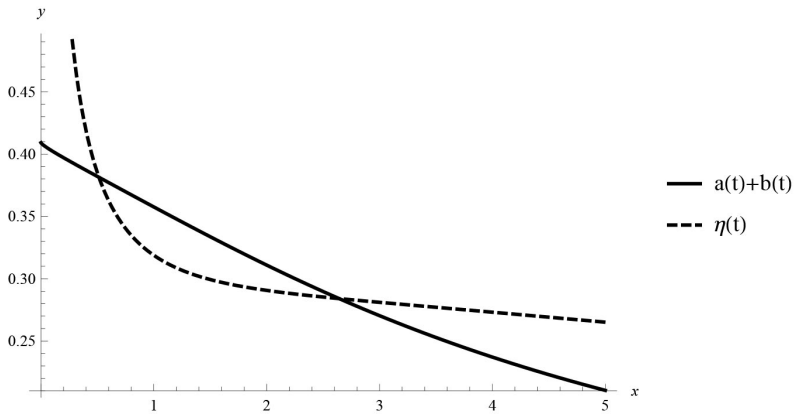

Figure 6: Example 3.26. Graph of the functions $a(t)+b(t)$ and $\eta(t)=c(t)+\sum_{j=1}^{3} \frac{d_{j}}{l_{j}^{2}}$ for $q=0.8$

Consider the function $p(t)=\left(E_{q}\left(-t^{q}\right)+0.1\right)$ and let the function $\phi \in C([-r, 0], \mathbb{R})$ and $t>0$ be such that $\sup _{\Theta \in[-r, 0]} p(t+\Theta)\|\phi(\Theta, .)\|_{\Delta}^{2}=p(t)\|\phi(0, .)\|_{\Delta}^{2}$, i.e., $\sup _{\Theta \in[-r, 0]} p(t+\Theta)|\phi(\Theta, x)|=p(t)|\phi(0, x)|, x \in$ $\Delta$.

Consider the following possible cases:

Case 1. Let $\phi(0, x)<0$ and $\phi(-r, x)<0$. Then from the inequality

$$
p(t) \sup _{\Theta \in[-r, 0]}|\phi(\Theta, x)| \leqslant \sup _{\Theta \in[-r, 0]} p(t+\Theta)|\phi(\Theta, x)|=p(t)|\phi(0, x)|, x \in \Delta
$$

we obtain $\phi(-r, x) \geqslant \phi(0, x)$ and from the monotonicity property of the function $f$ we get $f(\phi(-r, x)) \geqslant$ $f(\phi(0, x))$ and $b(t) f(\phi(-r, x)) \phi(0, x) \leqslant b(t) f(\phi(0, x)) \phi(0, x)$. Using inequalities $|f(\phi(0, x))|=-f(\phi(0, x)) \leqslant$ $|\phi(0, x)|=-\phi(0, x)$ (see Figure 5) and $b(t) \geqslant 0$ we get the inequalities $b(t) f(\phi(0, x)) \phi(0, x) \leqslant b(t) \phi^{2}(0, x)$ and

$$
a(t) f(\phi(0, x)) \phi(0, x)+b(t) f(\phi(-r, x)) \phi(0, x) \leqslant(a(t)+b(t)) \phi^{2}(0, x) .
$$

Case 2. Let $\phi(0, x)<0$ and $\phi(-r, x)>0$. Then $f(\phi(-r, x)) \geqslant 0$ and note $\mid f(\phi(-r, x) \mid=f(\phi(-r, x)) \geqslant$ $|\phi(-r, x)|=-\phi(0, x) \geqslant 0$ and $f(\phi(-r, x)) \phi(0, x) \leqslant-\phi^{2}(0, x) \leqslant 0$. Then we obtain

$a(t) f(\phi(0, x)) \phi(0, x)+b(t) f(\phi(-r, x)) \phi(0, x) \leqslant a(t) f(\phi(0, x)) \phi(0, x) \leqslant a(t) \phi^{2}(0, x) \leqslant(a(t)+b(t)) \phi^{2}(0, x)$.

Case 3. Let $\phi(0, x)>0$. Then $|\phi(-r, x)| \leqslant \phi(0, x)$ and from the monotonicity property of the function $f$ it follows that $f(\phi(-r, x)) \leqslant f(|\phi(-r, x)|) \leqslant f(\phi(0, x))$. Therefore,

$$
a(t) f(\phi(0, x)) \phi(0, x)+b(t) f(\phi(-r, x)) \phi(0, x) \leqslant(a(t)+b(t)) f(\phi(0, x)) \phi(0, x) \leqslant(a(t)+b(t)) \phi^{2}(0, x) .
$$


Therefore, the inequality

$$
a(t) \int_{\Delta} f(\phi(0, x)) \phi(0, x) d x+b(t) \int_{\Delta} f(\phi(-r, x)) \phi(0, x) d x \leqslant \xi(t)\|\phi(0, .)\|_{\Delta}^{2}
$$

holds, i.e., Assumption 3.23 is satisfied.

Then we get the inequality

$$
\begin{aligned}
-p(t) \eta(t)- & p(t) \sum_{j=1}^{3} \frac{d_{j}}{l_{j}^{2}}+p(t) \xi(t)+0.5{ }_{0}^{R L} D_{t}^{q} p(t) \\
= & -\left(E_{q}\left(-t^{q}\right)+0.1\right)\left(\frac{0.55}{t q \Gamma(1-q)\left(E_{q}\left(-t^{q}\right)+0.1\right)}+\frac{0.005}{E_{q}\left(-t^{q}\right)+0.1}+\sum_{j=1}^{3} \frac{d_{j}}{l_{j}^{2}}\right) \\
& +\left(E_{q}\left(-t^{q}\right)+0.1\right) \frac{0.45 E_{q}\left(-t^{q}\right)}{E_{q}\left(-t^{q}\right)+0.1}+0.5{ }_{0}^{R L} D_{t}^{q}\left(E_{q}\left(-t^{q}\right)+0.1\right) \\
\leqslant & -\frac{0.55}{t^{q} \Gamma(1-q)}-0.005+0.45 E_{q}\left(-t^{q}\right)+0.5\left(-E_{q}\left(-t^{q}\right)+\frac{1.1}{t^{q} \Gamma(1-q)}\right) \\
= & -\frac{0.55}{t^{q} \Gamma(1-q)}-0.005+0.45 E_{q}\left(-t^{q}\right)-0.5 E_{q}\left(-t^{q}\right)+\frac{0.55}{t^{q} \Gamma(1-q)} \\
= & -0.005-0.05 E_{q}\left(-t^{q}\right)=-0.05\left(E_{q}\left(-t^{q}\right)+0.1\right) \leqslant-0.005 .
\end{aligned}
$$

According to Theorem 3.24, the zero equilibrium point of scalar RDFrDNN (3.1) is uniformly stable.

Therefore, in the case the activation functions are not Lipschitz and the coefficients $c_{i}$ are not constants in RDFrDNN (3.1), we can use Lyapunov function depending directly on the time variable and its Caputo fractional Dini derivative is applicable to study the stability.

\section{Acknowledgment}

Research was partially supported by Fund MU17-FMI-007, University of Plovdiv Paisii Hilendarski.

\section{References}

[1] R. Agarwal, S. Hristova, D. O'Regan, Lyapunov functions and strict stability of Caputo fractional differential equations, Adv. Difference Equ., 2015 (2015), 20 pages. 1, 2.1

[2] R. Agarwal, S. Hristova, D. O'Regan, Lyapunov functions and stability of Caputo fractional differential equations with delays, (to be published). 2.13

[3] R. Agarwal, S. Hristova, D. O'Regan, A survey of Lyapunov functions, stability and impulsive Caputo fractional differential equations, Fract. Calc. Appl. Anal., 19 (2016), 290-318. 1, 2.1

[4] R. Agarwal, D. O'Regan, S. Hristova, Stability of Caputo fractional differential equations by Lyapunov functions, Appl. Math., 60 (2015), 653-676.

[5] R. Agarwal, D. O'Regan, S. Hristova, M. Cicek, Practical stability with respect to initial time difference for Caputo fractional differential equations, Commun. Nonlinear Sci. Numer. Simul., 42 (2017), 106-120. 1, 2.1

[6] H. Bao, J. H. Park, J. Cao, Synchronization of fractional-order complex-valued neural networks with time delay, Neural Netw., 81 (2016), 16-28. 1

[7] L. Chen, Y. Chai, R. Wu, T. Ma, H. Zhai, Dynamic analysis of a class of fractional-order neural networks with delay, Neurocomputing, 111 (2013), 190-194. 3.3

[8] B. Chen, J. Chen, Razumikhin-type stability theorems for functional fractional-order differential systems and applications, Appl. Math. Comput., 254 (2015), 63-69. 1, 2.12

[9] D. Chen, R. Zhang, X. Liu, X. Ma, Fractional order Lyapunov stability theorem and its applications in synchronization of complex dynamical networks, Commun. Nonlinear Sci. Numer. Simul., 19 (2014), 4105-4121. 1

[10] M. A. Duarte-Mermoud, N. Aguila-Camacho, J. A. Gallegos, R. Castro-Linares, Using general quadratic Lyapunov functions to prove Lyapunov uniform stability for fractional order systems, Commun. Nonlinear Sci. Numer. Simul., 22 (2015), 650-659. 1.2 
[11] Y. Huang, H. Zhang, Z. Wang, Dynamical stability analysis of multiple equilibrium points in time-varying delayed recurrent neural networks with discontinuous activation functions, Neurocomputing, 91 (2012), 21-28. 1

[12] R. Li, J. Cao, A. Alsaedi, F. Alsaadi, Stability analysis of fractional-order delayed neural networks, Nonlinear Anal. Model. Control, 22 (2017), 505-520. 3.2.1

[13] Y. Li, Y. Chen, I. Podlubny, Mittag-Leffler stability of fractional order nonlinear dynamic systems, Automatica J. IFAC, 45 (2009), 1965-1969. 1

[14] Y. Li, Y. Chen, I. Podlubny, Stability of fractional-order nonlinear dynamic systems: Lyapunov direct method and generalized Mittag-Leffler stability, Comput. Math. Appl., 59 (2010), 1810-1821. 1, 2.1

[15] C. Li, G. Feng, Delay-interval-dependent stability of recurrent neural networks with time-varying delay, Neurocomputing, 72 (2009), 1179-1183. 1

[16] J. Liang, J. Cao, Global Exponential Stability of Reaction-Diffusion Recurrent Neural Networks with Time-Varying Delays, Phys. Lett. A, 314 (2003), 434-442. 3.2

[17] X. Lou, B. Cui, Boundedness and Exponential Stability for Nonautonomous Cellular Neural Networks with ReactionDiffusion Terms, Chaos Solitons Fractals, 33 (2007), 653-662. 3.2

[18] C. M. Marcus, R. M. Westervelt, Stability of analog neural networks with delay, Phys. Rev. A, 39 (1989), $347-359.1$

[19] M. E. J. Newman, Communities, modules and large-scale structure in networks, Nature Phys., 8 (2012), 25-31. 1

[20] I. Podlubny, Fractional Differential Equations, Academic Press, San Diego, (1999). 1, 1

[21] A. Rahimi, B. Recht, Weighted sums of random kitchen sinks: Replacing minimization with randomization in learning, Adv. Neural Information Processing Syst., 2008 (2008), 1313-1320. 3.5

[22] S. J. Sadati, R. Ghaderi, A. Ranjbar, Some fractional comparison results and stability theorem for fractional time delay systems, Rom. Reports Phy., 65 (2013), 94-102. 2.1, 2.2, 2.10

[23] R. K. Saxena, A. M. Mathai, H. J. Haubold, Space-time Fractional Reaction-Diffusion Equations Associated with a Generalized Riemann-Liouville Fractional Derivative, Axioms, 3 (2014), 320-334. 1

[24] I. M. Stamova, On the Lyapunov theory for functional differential equations of fractional order, Proc. Amer. Math. Soc., 144 (2016), 1581-1593. 2.2

[25] I. M. Stamova, S. Simeonov, Delayed ReactionDiffusion Cellular Neural Networks of Fractional Order: MittagLeffler Stability and Synchronization, J. Comput. Nonlinear Dynam., 13 (2017), 7 pages. 2.2, 2.2, 3.2 .1

[26] I. Stamova, G. Stamov, Functional and Impulsive Differential Equations of Fractional Order: Qualitative Analysis and Applications, CRC Press, New York, (2016). 2.1, 2.2, 2.8

[27] J. Stigler, F. Ziegler, A. Gieseke, J. C. M. Gebhardt, M. Rief, The complex folding network of single calmodulin molecules, Science, 334 (2011), 512-516. 1

[28] J. Vasundhara Devi, F. A. Mc Rae, Z. Drici, Variational Lyapunov method for fractional differential equations, Comput. Math. Appl., 64 (2012), 2982-2989. 2.1

[29] D. Wanduku, G. S. Ladde, Global properties of a two-scale network stochastic delayed human epidemic dynamic model, Nonlinear Anal. Real World Appl., 13 (2012), 794-816. 1

[30] H. Wang, Y. Yu, G. Wen, Stability analysis of fractional-order Hopfield neural networks with time delays, Neural Netw., 55 (2014), 98-109. 1

[31] S. Wu, C. Li, X. Liao, S. Duan, Exponential stability of impulsive discrete systems with time delay and applications in stochastic neural networks: a Razumikhin approach, Neurocomputing, 82 (2012), 29-36. 1

[32] X. Yang, Q. Song, Y. Liu, Z. Zhao, Finite-time stability analysis of fractional-order neural networks with delay, Neurocomputing, 152 (2015), 19-26. 1

[33] W. Zhang, R. Wu, J. Cao, A. Alsaedi, T. Hayat, Synchronization of a class of fractional-order neural networks with multiple time delays by comparison principles, Nonlinear Anal. Model. Control, 22 (2017), 636-645. 1, 2.6, 3.2.1 